\title{
A Redox Isomerization Strategy for Accessing Modular Azobenzene Photoswitches with Near Quantitative Bidirectional Photoconversion
}

\author{
Supplemental Material
}

(42 pages)

Jie S. Zhu, Julio M. Larach, Robert J. Tombari, Phillip W. Gingrich, Stanley R. Bode, Jeremy R. Tuck, Hunter T. Warren, Jung-Ho Son, Whitney C. Duim, James C. Fettinger, Makhluf J. Haddadin, Dean J. Tantillo, Mark J. Kurth, ${ }^{*}$ David E. Olson ${ }^{*}$

\section{Contents}

Figure S1

Figure S2

1. General Information

2. Optimization of p-Formylazobenzene Synthesis

3. General Procedure for the Synthesis of p-Formylazobenzenes

4. General Procedure for the Synthesis of Modular Photoswitches

5. Experimental Procedure for Synthesizing 9

6. Experimental Procedures for Synthesizing 25

7. Constant Illumination NMR Experiments

8. UV-Vis Characterization for Compounds 8, 13, and 25

9. Photoswitchable Surfactant Experiments

10. Crystallography Information for 7, 8, and 19.

11. Computational Details

12. Supplemental References

13. Spectra 


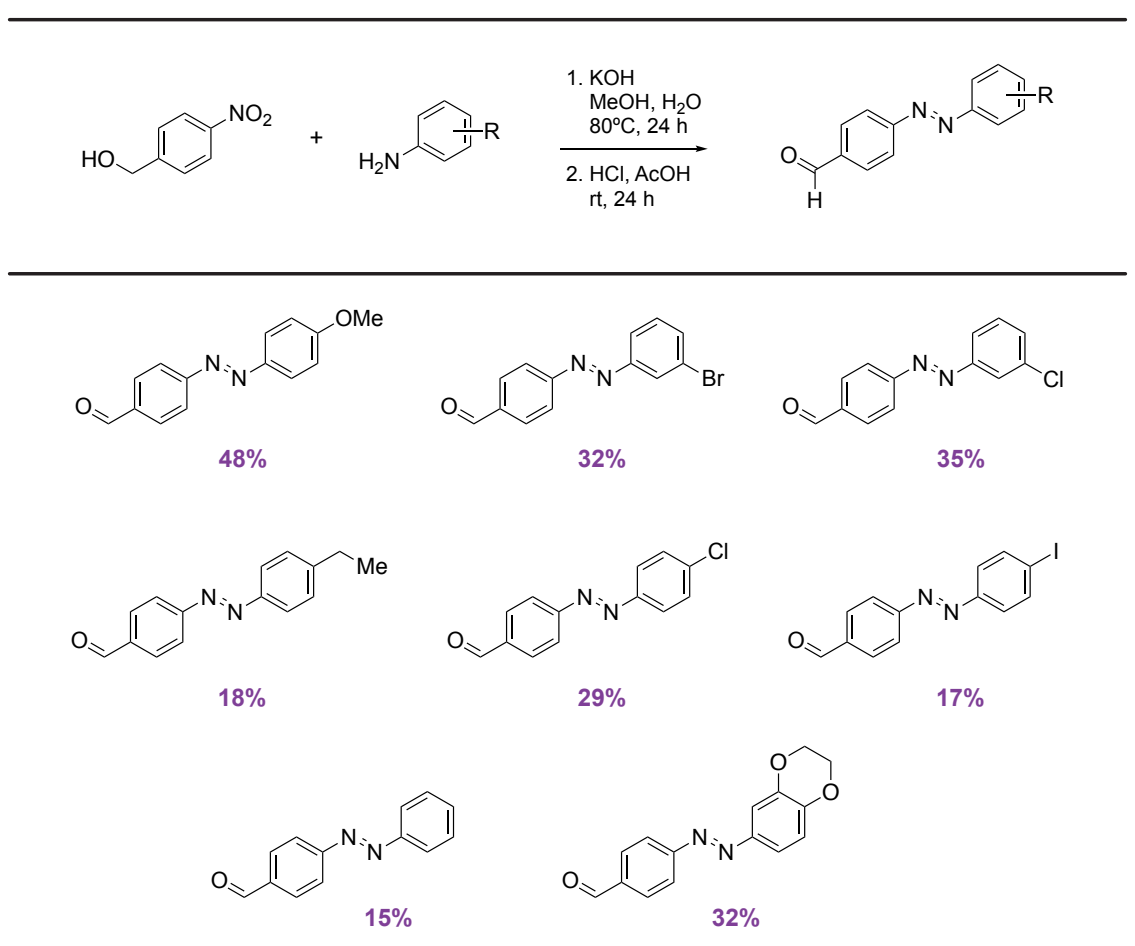

Figure S1. Synthesis of $p$-formylazobenzenes. Isolated yields are shown in purple. 

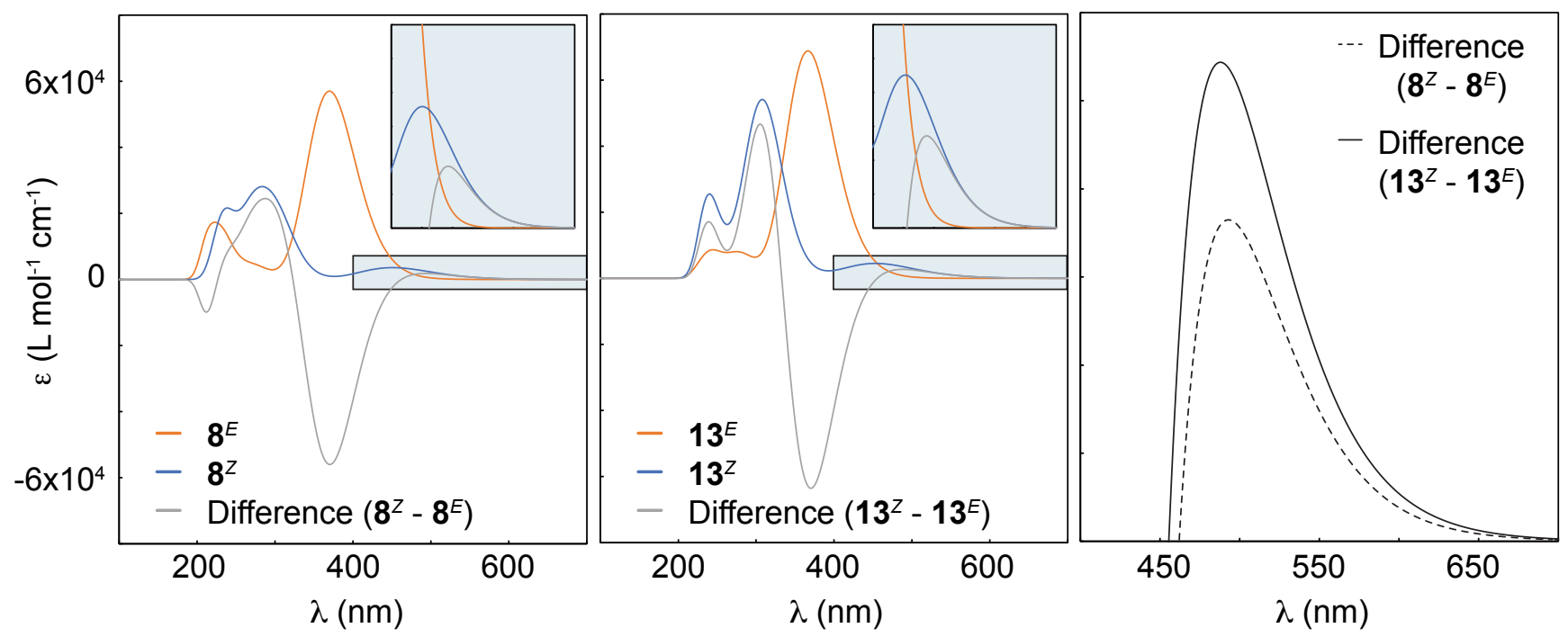

Figure S2. Calculated UV-Vis Spectra for the Isomers of $\mathbf{8}$ and 13. Calculations predict a greater difference between the spectra of the $\mathrm{E}$ and Z isomers for the modular photoswitch $\mathbf{1 3}$ as compared to the aldehyde 8 . 


\section{General Information}

All chemicals were purchased from standard commercial suppliers and used without further purification. Anhydrous solvents were dispensed from a solvent purification system utilizing dry neutral alumina or prepared using dry molecular sieves. Analytical TLC was performed using pre-coated plates (silica gel 60 F254) and visualized with UV light or an $\mathrm{I}_{2}$ chamber. Flash chromatography was performed using $60 \AA 230-400$ mesh silica gel (Fisher). ${ }^{1} \mathrm{H}$ NMR spectra and proton decoupled ${ }^{13} \mathrm{C}$ NMR spectra were obtained on a $400 \mathrm{MHz}$ Bruker, $600 \mathrm{MHz}$ Varian, or $800 \mathrm{MHz}$ Bruker NMR spectrometer. ${ }^{1} \mathrm{H}$ and ${ }^{13} \mathrm{C}$ chemical shifts $(\delta)$ are reported in parts per million (ppm) referenced to residual solvent peaks. Multiplicities are given as $s$ (singlet), $d$ (doublet), $t$ (triplet), q (quartet), and $\mathrm{m}$ (multiplet). Coupling constants $(J)$ are given in $\mathrm{Hz}$. For HRMS analysis, samples were analyzed by flow-injection analysis into a Thermo Fisher Scientific LTQ Orbitrap (San Jose, CA) operated in the centroided mode. Samples were injected into a mixture of $50 \% \mathrm{MeOH} / \mathrm{H}_{2} \mathrm{O}$ and $0.1 \%$ formic acid at a flow of $0.2 \mathrm{~mL} / \mathrm{min}$. Source parameters were $5.5 \mathrm{kV}$ spray voltage, capillary temperature of $275^{\circ} \mathrm{C}$, and sheath gas setting of 20. Spectral data were acquired at a resolution setting of 100,000 fwhm with the lockmass feature, which typically results in sufficient mass accuracy. Uncorrected melting point values were gathered using a Stanford Research Systems OptiMelt MPA100. LCMS analysis was carried out using a Waters 2695 equipped with an Ascentis Express C18, $2.7 \mu \mathrm{m}$ HPLC column using a gradient of $\mathrm{MeCN} / \mathrm{H}_{2} \mathrm{O}$ with $0.1 \%$ formic acid. The HLPC was also equipped with an inline Water 996 photodiode array detector operating between 250 and $800 \mathrm{~nm}$ and a Waters micromass $Z Q$ mass spectrometer in ESI+ mode.

\section{Optimization of $p$-Formylazobenzene Synthesis}

Optimization of the $p$-formylazobenzene synthesis began with two reactions differing in the choice of base employed. Sodium tert-butoxide was used in one reaction, while potassium hydroxide was used in the other. In both reactions, the base (10 equiv) was combined with 4-methoxyaniline (10 equiv), ethanol (3.0 $\mathrm{mL}$ ), and water $(3.5 \mathrm{~mL})$ in a microwave vial, which was sealed with an aluminum crimp cap. Each reaction was heated in an oil bath for $24 \mathrm{~h}$ at $100^{\circ} \mathrm{C}$. Upon cooling, the unpurified azo-imine was then hydrolyzed with concentrated hydrochloric acid $(20 \mathrm{~mL})$ and anhydrous acetic acid $(20 \mathrm{~mL})$ for $24 \mathrm{~h}$ at room temperature. After extraction with dichloromethane $(3 \times 50 \mathrm{~mL})$ and purification with flash column chromatography, each reaction yielded the targeted $p$-formylazobenzene $(42 \%$ and $43 \%$, respectively; entries 1 and 2$)$. The optimization process continued by screening different solvents, such as ethylene glycol, ethanol, isopropanol and dimethylformamide (entries 3, 4, $5 \& 6$ ), but none of these improved product yield. Therefore, we continued our investigation using methanol as the solvent and potassium hydroxide as the base. Different amounts of base were evaluated next, ranging from 5 to 20 equivalents. The results showed that 5 and 10 equivalents gave the best yields (entries $8 \& 2$ ). Next, we decreased the amount of 4-methoxyaniline to 5 equivalents, but this proved detrimental (entries $7 \& 9$ ). Additionally, varying amounts of methanol and water were also screened (entries 12, 13, \& 14), but each modification decreased product yield. Methanol and water in a $\sim 1: 1$ ratio was optimal for product formation. Lastly, various temperatures $\left(40{ }^{\circ} \mathrm{C}, 60{ }^{\circ} \mathrm{C}\right.$, and $\left.80{ }^{\circ} \mathrm{C}\right)$ were evaluated. Lower temperatures led to reduced yields (entries $15 \& 16$ ) while elevated temperatures gave more optimal yields (entries $3 \& 17$ ). 

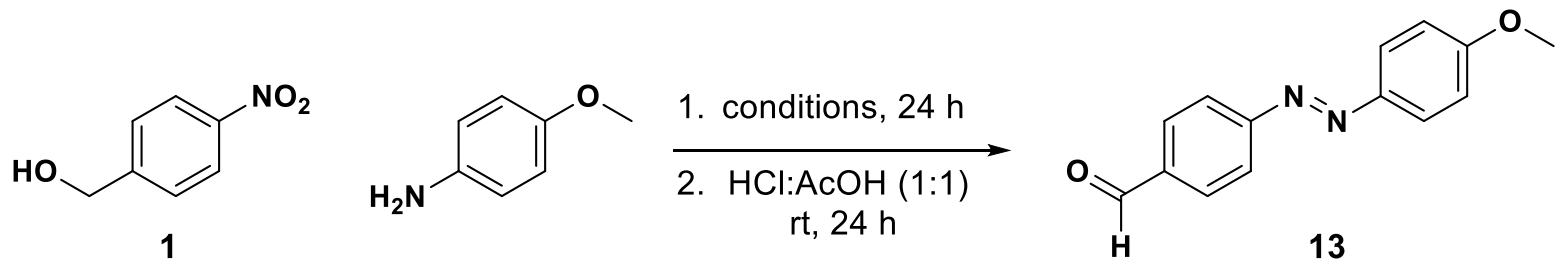

\begin{tabular}{|c|c|c|c|c|c|c|c|}
\hline entry & base & base eq & aniline eq & temperature & solvent & water & yield \\
\hline 1 & $\mathrm{NaO}{ }^{t} \mathrm{bu}$ & 10 & 10 & 100 & $3 \mathrm{~mL} \mathrm{MeOH}$ & $3.5 \mathrm{~mL}$ & $42 \%$ \\
\hline 2 & $\mathrm{KOH}$ & 10 & 10 & 100 & $3 \mathrm{~mL} \mathrm{MeOH}$ & $3.5 \mathrm{~mL}$ & $43 \%$ \\
\hline 3 & $\mathrm{NaO}{ }^{t} \mathrm{bu}$ & 10 & 10 & 100 & $3 \mathrm{~mL}\left(\mathrm{CH}_{2} \mathrm{OH}\right)_{2}$ & $3.5 \mathrm{~mL}$ & $16 \%$ \\
\hline 4 & $\mathrm{NaO}{ }^{t} \mathrm{bu}$ & 10 & 10 & 100 & $3 \mathrm{~mL} \mathrm{EtOH}$ & $3.5 \mathrm{~mL}$ & $39 \%$ \\
\hline 5 & $\mathrm{NaO}{ }^{t} \mathrm{bu}$ & 10 & 10 & 100 & $3 \mathrm{~mL} \mathrm{iPrOH}$ & $3.5 \mathrm{~mL}$ & $19 \%$ \\
\hline 6 & $\mathrm{NaO}{ }^{t} \mathrm{bu}$ & 10 & 10 & 100 & $3 \mathrm{~mL}$ DMF & $3.5 \mathrm{~mL}$ & $0 \%$ \\
\hline 7 & $\mathrm{KOH}$ & 5 & 5 & 100 & $3 \mathrm{~mL} \mathrm{MeOH}$ & $3.5 \mathrm{~mL}$ & $24 \%$ \\
\hline 8 & $\mathrm{KOH}$ & 5 & 10 & 100 & $3 \mathrm{~mL} \mathrm{MeOH}$ & $3.5 \mathrm{~mL}$ & $45 \%$ \\
\hline 9 & $\mathrm{KOH}$ & 10 & 5 & 100 & $3 \mathrm{~mL} \mathrm{MeOH}$ & $3.5 \mathrm{~mL}$ & $29 \%$ \\
\hline 10 & $\mathrm{KOH}$ & 15 & 10 & 100 & $3 \mathrm{~mL} \mathrm{MeOH}$ & $3.5 \mathrm{~mL}$ & $41 \%$ \\
\hline 11 & $\mathrm{KOH}$ & 20 & 10 & 100 & $3 \mathrm{~mL} \mathrm{MeOH}$ & $3.5 \mathrm{~mL}$ & $35 \%$ \\
\hline 12 & $\mathrm{KOH}$ & 10 & 10 & 100 & $5 \mathrm{~mL} \mathrm{MeOH}$ & $1.5 \mathrm{~mL}$ & $33 \%$ \\
\hline 13 & $\mathrm{KOH}$ & 10 & 10 & 100 & $6.5 \mathrm{~mL} \mathrm{MeOH}$ & $0 \mathrm{~mL}$ & $23 \%$ \\
\hline 14 & $\mathrm{KOH}$ & 10 & 10 & 100 & $1.5 \mathrm{~mL} \mathrm{MeOH}$ & $5 \mathrm{~mL}$ & $42 \%$ \\
\hline 15 & $\mathrm{KOH}$ & 10 & 10 & 40 & $3 \mathrm{~mL} \mathrm{MeOH}$ & $3.5 \mathrm{~mL}$ & $6 \%$ \\
\hline 16 & $\mathrm{KOH}$ & 10 & 10 & 60 & $3 \mathrm{~mL} \mathrm{MeOH}$ & $3.5 \mathrm{~mL}$ & $33 \%$ \\
\hline 17 & $\mathrm{KOH}$ & 10 & 10 & 80 & $3 \mathrm{~mL} \mathrm{MeOH}$ & $3.5 \mathrm{~mL}$ & $48 \%$ \\
\hline
\end{tabular}

\section{General Procedure for the Synthesis of $p$-Formylazobenzenes}<smiles>O=[N+]([O-])c1ccc(CO)cc1</smiles><smiles>[R]c1ccc(N)cc1</smiles>

1. $\mathrm{KOH}$ (10 equiv) $\mathrm{MeOH}, \mathrm{H}_{2} \mathrm{O}$ $100^{\circ} \mathrm{C}, 24 \mathrm{~h}$

2. $\mathrm{HCl}: \mathrm{AcOH}(1: 1)$ rt, $24 \mathrm{~h}$<smiles>[R]c1ccc(/N=N/c2ccc(C=O)cc2)cc1</smiles>

A Biotage microwave vial was charged with a stir bar, 4-nitrobenzyl alcohol 1 (0.5 mmol, 1 equiv), an appropriate aniline (5 mmol, 10 equiv), potassium hydroxide (5 mmol, 10 equiv), methanol $(3.0 \mathrm{~mL})$ and water $(3.5 \mathrm{~mL})$, and the vial was sealed with an aluminum crimp cap. The reaction mixture was stirred and heated at $100^{\circ} \mathrm{C}$ for $24 \mathrm{~h}$ in an oil bath. The reaction mixture was then cooled to room temperature. The solid was collected by vacuum filtration, washed with cold methanol, dissolved in dichloromethane, and transferred to a round bottom flask. Concentrated hydrochloric acid $(20 \mathrm{~mL})$ and anhydrous acetic acid $(20 \mathrm{~mL})$ were added to the flask, and the reaction was stirred for $24 \mathrm{~h}$ at room temperature. The mixture was then transferred to a separatory funnel with water $(100 \mathrm{~mL})$ and extracted with dichloromethane $(3 \times 50 \mathrm{~mL})$. The organic layers 
were combined, dried with magnesium sulfate, and filtered. Dichloromethane was removed by rotatory evaporation. The solid was purified by flash column chromatography on silica gel (gradient elution: 19:1 $\rightarrow 1: 19$ hexanes:EtOAc).<smiles>COc1ccc(/N=N/c2ccc(C=O)cc2)cc1</smiles>

\section{8}

(E)-4-((4-methoxyphenyl)diazenyl)benzaldehyde

Yield: $58 \mathrm{mg}(48 \%)$. Scaled up to $2.5 \mathrm{mmol}$ scale: $283 \mathrm{mg} \mathrm{(46 \% ).}$

Physical property: Orange solid.

MP: $69-70{ }^{\circ} \mathrm{C}$.

${ }^{1} \mathrm{H}$ NMR $\left(400 \mathrm{MHz}, \mathrm{CDCl}_{3} \delta 10.09(\mathrm{~s}, 1 \mathrm{H}), 8.03-7.94(\mathrm{~m}, 6 \mathrm{H}), 7.04(\mathrm{~d}, \mathrm{~J}=8.7,2 \mathrm{H}), 3.91(\mathrm{~s}, 3 \mathrm{H})\right.$.

${ }^{13} \mathrm{C}$ NMR (101 MHz, DMSO-d6) $\delta 193.1,163.3,155.7,146.8,137.5,131.2,125.6,123.3,115.3,56.2$.

HRMS (Orbitrap): Calcd for $\left[\mathrm{C}_{14} \mathrm{H}_{13} \mathrm{~N}_{2} \mathrm{O}_{2}{ }^{+}, \mathrm{M}+\mathrm{H}\right]^{+}: 241.0977$, found: 241.0962 .<smiles>O=Cc1ccc(N=Nc2cccc(Br)c2)cc1</smiles>

\section{(E)-4-((3-bromophenyl)diazenyl)benzaldehyde}

Yield: $47 \mathrm{mg} \mathrm{(32 \% )}$.

Physical property: Orange sticky powder.

${ }^{1} \mathrm{H}$ NMR $\left(400 \mathrm{MHz}, \mathrm{CDCl}_{3}\right) \delta 10.12(\mathrm{~s}, 1 \mathrm{H}), 8.10(\mathrm{~s}, 1 \mathrm{H}), 8.08-8.02(\mathrm{~m}, 4 \mathrm{H}), 7.93(\mathrm{~d}, \mathrm{~J}=7.8,1 \mathrm{H}), 7.65(\mathrm{~d}$, $J=7.9,1 \mathrm{H}), 7.44(\mathrm{t}, J=7.8,1.7,1 \mathrm{H}) \mathrm{ppm}$

${ }^{13} \mathrm{C}$ NMR $\left(101 \mathrm{MHz}, \mathrm{CDCl}_{3}\right) \delta 191.5,155.5,153.3,137.8,134.5,130.7,130.6,124.9,123.5,123.4,123.3 \mathrm{ppm}$ HRMS (Orbitrap): Calcd for $\left[\mathrm{C}_{13} \mathrm{H}_{10} \mathrm{BrN}_{2} \mathrm{O}^{+}, \mathrm{M}+\mathrm{H}\right]^{+}: 288.9977$, found: 288.9966<smiles>O=Cc1ccc(/N=N/c2cccc(Cl)c2)cc1</smiles>

\section{(E)-4-((3-chlorophenyl)diazenyl)benzaldehyde}

Yield: $43 \mathrm{mg} \mathrm{(35 \% ).}$

Physical property: Orange sticky powder.

${ }^{1} \mathrm{H}$ NMR $\left(400 \mathrm{MHz}, \mathrm{CDCl}_{3}\right) \delta 10.12(\mathrm{~s}, 1 \mathrm{H}), 8.07-8.04(\mathrm{~m}, 4 \mathrm{H}), 7.94(\mathrm{~s}, 1 \mathrm{H}), 7.91-7.85(\mathrm{~m}, 1 \mathrm{H}), 7.52-7.48$ $(\mathrm{m}, 2 \mathrm{H}) \mathrm{ppm}$

${ }^{13} \mathrm{C}$ NMR $\left(101 \mathrm{MHz}, \mathrm{CDCl}_{3}\right) \delta 191.5,155.5,153.3,137.8,135.4,131.6,130.7,130.3,123.5,122.9,122.0 \mathrm{ppm}$ HRMS (Orbitrap): Calcd for $\left[\mathrm{C}_{13} \mathrm{H}_{10} \mathrm{CIN}_{2} \mathrm{O}+, \mathrm{M}+\mathrm{H}\right]^{+}: 245.0482$, found: 245.0465 
<smiles>CCc1ccc(/N=N/c2ccc(C=O)cc2)cc1</smiles>

\section{(E)-4-((4-ethylphenyl)diazenyl)benzaldehyde}

Yield: $21 \mathrm{mg}(18 \%)$.

Physical property: Orange sticky powder.

${ }^{1} \mathrm{H}$ NMR $\left(400 \mathrm{MHz}, \mathrm{CDCl}_{3}\right) \delta 10.10(\mathrm{~s}, 1 \mathrm{H}), 8.08-7.99(\mathrm{~m}, 4 \mathrm{H}), 7.90(\mathrm{~d}, J=7.7,2 \mathrm{H}), 7.37(\mathrm{~d}, J=7.9,2 \mathrm{H}), 2.75$ (q, J=7.6, 2H), $1.31(\mathrm{t}, J=7.6,3 \mathrm{H}) \mathrm{ppm}$

${ }^{13} \mathrm{C}$ NMR $\left(101 \mathrm{MHz}, \mathrm{CDCl}_{3}\right) \delta 191.6,156.0,150.9,149.1,137.2,130.7,128.7,123.4,123.2,28.9,15.3$ ppm HRMS (Orbitrap): Calcd for $\left[\mathrm{C}_{15} \mathrm{H}_{15} \mathrm{~N}_{2} \mathrm{O}^{+}, \mathrm{M}+\mathrm{H}\right]^{+}: 239.1184$, found: 239.1169<smiles>O=Cc1ccc(/N=N/c2ccc(Cl)cc2)cc1</smiles>

\section{(E)-4-((4-chlorophenyl)diazenyl)benzaldehyde}

Yield: $35 \mathrm{mg}(29 \%)$.

Physical property: Orange sticky powder.

${ }^{1} \mathrm{H}$ NMR $\left(400 \mathrm{MHz}, \mathrm{CDCl}_{3}\right) \delta 10.11(\mathrm{~s}, 1 \mathrm{H}), 8.08-8.00(\mathrm{~m}, 4 \mathrm{H}), 7.90(\mathrm{~d}, \mathrm{~J}=8.1,2 \mathrm{H}), 7.69(\mathrm{~d}, \mathrm{~J}=8.1,2 \mathrm{H}) \mathrm{ppm}$

${ }^{13} \mathrm{C}$ NMR $\left(101 \mathrm{MHz}, \mathrm{CDCl}_{3}\right) \delta 191.6,155.7,150.9,138.1,137.6,130.7,129.5,124.5,123.4 \mathrm{ppm}$

HRMS (Orbitrap): Calcd for $\left[\mathrm{C}_{13} \mathrm{H}_{10} \mathrm{CIN}_{2} \mathrm{O}^{+}, \mathrm{M}+\mathrm{H}\right]^{+}:$:245.0482, found: 245.0466<smiles>O=Cc1ccc(/N=N/c2ccc(I)cc2)cc1</smiles>

\section{(E)-4-((4-iodophenyl)diazenyl)benzaldehyde}

Yield: $28 \mathrm{mg}(17 \%)$.

Physical property: Orange sticky powder.

${ }^{1} \mathrm{H}$ NMR $\left(400 \mathrm{MHz}, \mathrm{CDCl}_{3}\right) \delta 10.11(\mathrm{~s}, 1 \mathrm{H}), 8.08-8.00(\mathrm{~m}, 4 \mathrm{H}), 7.90(\mathrm{~d}, J=8.2,2 \mathrm{H}), 7.69(\mathrm{~d}, \mathrm{~J}=8.2,2 \mathrm{H}) \mathrm{ppm}$

${ }^{13} \mathrm{C}$ NMR $\left(101 \mathrm{MHz}, \mathrm{CDCl}_{3}\right) \delta 191.5,155.6,151.8,138.6,137.7,130.7,124.8,123.4,99.1 \mathrm{ppm}$

HRMS (Orbitrap): Calcd for $\left[\mathrm{C}_{13} \mathrm{H}_{10} \mathrm{IN}_{2} \mathrm{O}^{+}, \mathrm{M}+\mathrm{H}\right]^{+}: 336.9838$, found: 336.9831<smiles>O=Cc1ccc(/N=N/c2ccccc2)cc1</smiles>

\section{(E)-4-(phenyldiazenyl)benzaldehyde}

Yield: $20 \mathrm{mg}(15 \%)$

Physical property: Orange sticky powder.

${ }^{1} \mathrm{H}$ NMR $\left(400 \mathrm{MHz}, \mathrm{C}_{6} \mathrm{D}_{6}\right) \delta 10.11(\mathrm{~s}, 1 \mathrm{H}), 8.06-8.03(\mathrm{~m}, 4 \mathrm{H}), 7.97(\mathrm{~d}, \mathrm{~J}=7.2,2 \mathrm{H}), 7.58-7.51(\mathrm{~m}, 3 \mathrm{H}) \mathrm{ppm}$ 
${ }^{13} \mathrm{C}$ NMR (101 MHz, $\left.\mathrm{CDCl}_{3}\right) \delta 191.6,155.9,152.5,137.4,132.0,130.7,129.2,123.3,123.3 \mathrm{ppm}$

HRMS (Orbitrap): Calcd for $\left[\mathrm{C}_{13} \mathrm{H}_{11} \mathrm{~N}_{2} \mathrm{O}^{+}, \mathrm{M}+\mathrm{H}\right]^{+}: 211.0871$, found: 211.0855<smiles>O=Cc1ccc(/N=N/c2ccc3c(c2)OCCO3)cc1</smiles>

\section{$(E)-4-((2,3-d i h y d r o b e n z o[b][1,4]$ dioxin-6-yl)diazenyl)benzaldehyde}

Yield: $19 \mathrm{mg}(14 \%)$.

Physical property: Orange sticky powder.

${ }^{1} \mathrm{H}$ NMR $\left(400 \mathrm{MHz}, \mathrm{CDCl}_{3}\right) \delta 10.09(\mathrm{~s}, 1 \mathrm{H}), 8.04-7.96(\mathrm{~m}, 4 \mathrm{H}), 7.57(\mathrm{~d}, \mathrm{~J}=8.7,1 \mathrm{H}), 7.53(\mathrm{~s}, 1 \mathrm{H}), 7.01(\mathrm{~d}$, $J=8.6,1.5,1 \mathrm{H}), 4.37-4.30(\mathrm{~m}, 4 \mathrm{H}) \mathrm{ppm}$

${ }^{13} \mathrm{C}$ NMR $\left(101 \mathrm{MHz}, \mathrm{CDCl}_{3}\right) \delta 191.7,156.0,147.4,147.4,144.0,137.1,130.7,123.1,119.8,119.1,117.6$, 111.0, 64.7, $64.2 \mathrm{ppm}$

HRMS (Orbitrap): Calcd for $\left[\mathrm{C}_{15} \mathrm{H}_{13} \mathrm{~N}_{2} \mathrm{O}_{3}{ }^{+}, \mathrm{M}+\mathrm{H}\right]^{+}: 269.0926$, found: 269.0912

\section{General Procedure for the Synthesis of Modular Photoswitches}<smiles>COc1ccc(/N=N/c2ccc(C=O)cc2)cc1</smiles><smiles>[R]C(=O)NN</smiles><smiles>[R]C(=O)N/N=C/c1ccc(/N=N/c2ccc(OC)cc2)cc1</smiles>

A Biotage microwave vial was charged with a stir bar, $13(0.5 \mathrm{mmol})$, an appropriate hydrazide $(0.5 \mathrm{mmol})$, ethanol $(5 \mathrm{~mL})$ and catalytic amount of trifluoroacetic acid $(10 \mu \mathrm{L})$. The vial was sealed with an aluminum crimp cap. The reaction mixture was stirred and heated in an $80^{\circ} \mathrm{C}$ oil bath until all reactants went into solution (typically 10 to 20 minutes). The reaction was then allowed to cool to room temperature, at which time the product precipitated. The reaction vial was placed in the freezer at $-20{ }^{\circ} \mathrm{C}$ overnight to further promote product precipitation. The solid was collected by vacuum filtration and washed with cold ethanol.<smiles>COc1ccc(/N=N/c2ccc(/C=N/NC(=O)c3ccccc3)cc2)cc1</smiles>

13

N'-((E)-4-((E)-(4-methoxyphenyl)diazenyl)benzylidene)benzohydrazide

Yield: $136 \mathrm{mg} \mathrm{(76 \% );} 299 \mathrm{mg}(83 \%)$ on a $1.0 \mathrm{mmol}$ scale.

Physical property: Orange sticky powder. 
${ }^{1} \mathrm{H}$ NMR $\left(400 \mathrm{MHz}\right.$, DMSO- $\left.d_{6}\right) \delta 11.99(\mathrm{~s}, 1 \mathrm{H}), 8.54(\mathrm{~s}, 1 \mathrm{H}), 8.03-7.89(\mathrm{~m}, 8 \mathrm{H}), 7.64-7.58(\mathrm{~m}, 1 \mathrm{H}), 7.55(\mathrm{t}$, $J=7.3,2 \mathrm{H}), 7.15(\mathrm{~d}, J=8.6,2 \mathrm{H}), 3.88(\mathrm{~s}, 3 \mathrm{H}) \mathrm{ppm}$

${ }^{13} \mathrm{C}$ NMR $\left(101 \mathrm{MHz}\right.$, DMSO- $\left.d_{6}\right) \delta 163.7,162.7,153.1,147.2,146.8,136.9,133.8,132.3,129.0,128.6,128.2$, 125.3, 123.0, 115.2, $56.2 \mathrm{ppm}$

HRMS (Orbitrap): Calcd for $\left[\mathrm{C}_{21} \mathrm{H}_{19} \mathrm{~N}_{4} \mathrm{O}_{2}{ }^{+}, \mathrm{M}+\mathrm{H}\right]^{+}: 359.1508$, found: 359.1507<smiles>COc1ccc(/N=N/c2ccc(/C=N/NC(=O)c3ccc4ccccc4c3)cc2)cc1</smiles>

14

$N^{\prime}-((E)-4-((E)-(4-m e t h o x y p h e n y l)$ diazenyl)benzylidene)-2-naphthohydrazide

Yield: $53 \mathrm{mg}(65 \%)$.

Physical property: Orange sticky powder.

${ }^{1} \mathrm{H}$ NMR $\left(400 \mathrm{MHz}\right.$, DMSO- $\left.d_{6}\right) \delta 12.18(\mathrm{~s}, 1 \mathrm{H}), 8.62-8.54(\mathrm{~m}, 2 \mathrm{H}), 8.08(\mathrm{t}, J=9.2,2 \mathrm{H}), 8.04-7.99(\mathrm{~m}, 2 \mathrm{H})$, $7.99-7.88(\mathrm{~m}, 6 \mathrm{H}), 7.70-7.59(\mathrm{~m}, 2 \mathrm{H}), 7.15(\mathrm{~d}, \mathrm{~J}=8.5,2 \mathrm{H}), 3.88(\mathrm{~s}, 3 \mathrm{H}) \mathrm{ppm}$

${ }^{13} \mathrm{C}$ NMR $\left(101 \mathrm{MHz}, \mathrm{DMSO}-d_{6}\right) \delta 163.7,162.7,153.1,147.2,146.8,136.9,134.9,132.5,131.1,129.4,128.6$, $128.4,128.2,127.4,125.2,124.8,123.3,115.2,56.2 \mathrm{ppm}$

HRMS (Orbitrap): Calcd for $\left[\mathrm{C}_{25} \mathrm{H}_{21} \mathrm{~N}_{4} \mathrm{O}_{2}{ }^{+}, \mathrm{M}+\mathrm{H}\right]^{+}: 409.1665$, found: 409.1654<smiles>COc1ccc(/N=N/c2ccc(/C=N/NC(=O)c3ccc(C)cc3)cc2)cc1</smiles>

15

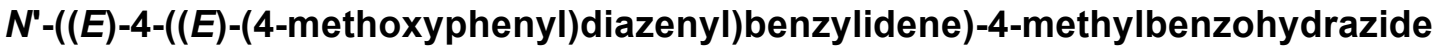

Yield: $58.6 \mathrm{mg}(79 \%)$.

Physical property: Yellow sticky powder.

${ }^{1} \mathrm{H}$ NMR $(400 \mathrm{MHz}$, DMSO-d $\left.)\right) \delta 11.92(\mathrm{~s}, 1 \mathrm{H}), 8.53(\mathrm{~s}, 1 \mathrm{H}), 7.98-7.87(\mathrm{~m}, 6 \mathrm{H}), 7.86(\mathrm{~d}, \mathrm{~J}=7.8,2 \mathrm{H}), 7.35(\mathrm{~d}$, $J=8.8,2 \mathrm{H}), 7.15(\mathrm{~d}, J=8.8,2 \mathrm{H}), 3.87(\mathrm{~s}, 3 \mathrm{H}), 2.39(\mathrm{~s}, 3 \mathrm{H}) \mathrm{ppm}$

${ }^{13} \mathrm{C}$ NMR $\left(101 \mathrm{MHz}\right.$, DMSO- $\left.d_{6}\right) \delta 163.5,162.7,153.0,146.9,146.8,142.4,137.0,130.9,129.5,128.5,128.2$, $125.2,123.8,123.30,120.32,115.2,56.2,55.8,21.5 \mathrm{ppm}$

HRMS (Orbitrap): Calcd for $\left[\mathrm{C}_{22} \mathrm{H}_{21} \mathrm{~N}_{4} \mathrm{O}_{2}{ }^{+}, \mathrm{M}+\mathrm{H}\right]^{+}: 373.1665$, found: 373.1663<smiles>COc1ccc(/N=N/c2ccc(/C=N/NC(=O)c3ccc(Br)cc3)cc2)cc1</smiles>

16

4-bromo- $N^{\prime}-((E)-4-((E)-(4-m e t h o x y p h e n y l)$ diazenyl)benzylidene)benzohydrazide

Yield: $68 \mathrm{mg}$ (88\%).

Physical property: Yellow sticky powder. 
${ }^{1} \mathrm{H}$ NMR $\left(400 \mathrm{MHz}\right.$, DMSO- $\left.d_{6}\right) \delta 12.05(\mathrm{~s}, 1 \mathrm{H}), 8.52(\mathrm{~s}, 1 \mathrm{H}), 8.00-7.83(\mathrm{~m}, 8 \mathrm{H}), 7.77(\mathrm{~d}, J=7.8,2 \mathrm{H}), 7.15(\mathrm{~d}$, $J=8.4,2 \mathrm{H}), 3.88(\mathrm{~s}, 3 \mathrm{H}) \mathrm{ppm}$

${ }^{13} \mathrm{C}$ NMR $\left(101 \mathrm{MHz}\right.$, DMSO- $\left.d_{6}\right) \delta 162.8,153.2,147.5,146.8,136.8,132.8,132.0,130.2,128.6,126.1,125.2$, 123.3, 120.3, 115.2, $56.2 \mathrm{ppm}$

HRMS (Orbitrap): Calcd for $\left[\mathrm{C}_{21} \mathrm{H}_{18} \mathrm{BrN}_{4} \mathrm{O}_{2}{ }^{+}, \mathrm{M}+\mathrm{H}\right]^{+}: 437.0613$, found: 437.0614<smiles>COc1ccc(/N=N/c2ccc(/C=N/NC(=O)c3ccc([N+](=O)[O-])cc3)cc2)cc1</smiles>

17

$N^{\prime}-((E)-4-((E)-(4-m e t h o x y p h e n y l)$ diazenyl)benzylidene)-4-nitrobenzohydrazide

Yield: $76.8 \mathrm{mg}(95 \%)$.

Physical property: Yellow sticky powder.

${ }^{1} \mathrm{H}$ NMR $\left.(400 \mathrm{MHz} \text {, DMSO-d })_{6}\right) 12.26(\mathrm{~s}, 1 \mathrm{H}), 8.54(\mathrm{~s}, 1 \mathrm{H}), 8.38(\mathrm{~d}, \mathrm{~J}=8.2,2 \mathrm{H}), 8.17(\mathrm{~d}, \mathrm{~J}=8.3,2 \mathrm{H}), 7.96-$

$7.88(\mathrm{~m}, 7 \mathrm{H}), 7.14(\mathrm{~d}, \mathrm{~J}=8.4,3 \mathrm{H}), 3.87(\mathrm{~s}, 4 \mathrm{H}) \mathrm{ppm}$

${ }^{13}$ C NMR $\left(101 \mathrm{MHz}\right.$, DMSO- $\left.d_{6}\right) \delta 162.8,162.0,153.2,149.8,148.3,146.8,139.4,136.6,129.7,128.8,125.2$, $124.1,123.3,115.2,56.2 \mathrm{ppm}$

HRMS (Orbitrap): Calcd for $\left[\mathrm{C}_{21} \mathrm{H}_{18} \mathrm{~N}_{5} \mathrm{O}_{4}{ }^{+}, \mathrm{M}+\mathrm{H}\right]^{+}: 404.1359$, found: 404.1346<smiles>COc1ccc(/N=N/c2ccc(/C=N/NC(=O)c3ccc(C(F)(F)F)cc3)cc2)cc1</smiles>

18

N'-((E)-4-((E)-(4-methoxyphenyl)diazenyl)benzylidene)-4-(trifluoromethyl)benzohydrazide

Yield: $63.9 \mathrm{mg}(75 \%)$.

Physical property: Yellow sticky powder.

${ }^{1} \mathrm{H}$ NMR $\left(400 \mathrm{MHz}, \mathrm{DMSO}-\mathrm{d}_{6}\right) \delta 12.18(\mathrm{~s}, 1 \mathrm{H}), 8.54(\mathrm{~s}, 1 \mathrm{H}), 8.14(\mathrm{~d}, \mathrm{~J}=7.9,2 \mathrm{H}), 8.00-7.82(\mathrm{~m}, 8 \mathrm{H}), 7.15(\mathrm{~d}$, $J=8.5,2 \mathrm{H}), 3.87(\mathrm{~s}, 3 \mathrm{H}) \mathrm{ppm}$

${ }^{13} \mathrm{C}$ NMR $\left(101 \mathrm{MHz}\right.$, DMSO- $\left.d_{6}\right) \delta 162.8,162.5,153.2,148.0,146.8,137.6,136.7,132.2,131.9,129.1,128.7$, $126.0,126.0,125.2,123.8,123.3,123.0,120.3,115.2,56.2 \mathrm{ppm}$

HRMS (Orbitrap): Calcd for $\left[\mathrm{C}_{22} \mathrm{H}_{18} \mathrm{~F}_{3} \mathrm{~N}_{4} \mathrm{O}_{2}{ }^{+}, \mathrm{M}+\mathrm{H}\right]^{+}: 427.1382$, found: 427.1375<smiles>COc1ccc(/N=N/c2ccc(/C=N/NC(=O)c3cccc(OC)c3)cc2)cc1</smiles>

19

3-methoxy-N'-((E)-4-((E)-(4-methoxyphenyl)diazenyl)benzylidene)benzohydrazide

Yield: $161 \mathrm{mg}(83 \%)$.

Physical property: Orange solid. 
MP: $219-223^{\circ} \mathrm{C}$.

${ }^{1} \mathrm{H}$ NMR $\left(400 \mathrm{MHz}\right.$, DMSO- $\left.d_{6}\right) \delta 11.95(\mathrm{~s}, 1 \mathrm{H}), 8.54(\mathrm{~s}, 1 \mathrm{H}), 8.05-7.88(\mathrm{~m}, 6 \mathrm{H}), 7.55-7.42(\mathrm{~m}, 3 \mathrm{H}), 7.22-$ $7.12(\mathrm{~m}, 3 \mathrm{H}), 3.88(\mathrm{~s}, 3 \mathrm{H}), 3.84(\mathrm{~s}, 3 \mathrm{H}) \mathrm{ppm}$

${ }^{13} \mathrm{C}$ NMR $\left(101 \mathrm{MHz}\right.$, DMSO- $\left.d_{6}\right) \delta 163.4,162.7,159.7,153.1,147.3,146.8,136.9,135.2,130.2,128.6,125.2$, 123.3, 120.3, 118.1, 115.2, 113.4, 56.2, $55.8 \mathrm{ppm}$

HRMS (Orbitrap): Calcd for $\left[\mathrm{C}_{22} \mathrm{H}_{21} \mathrm{~N}_{4} \mathrm{O}_{3}{ }^{+}, \mathrm{M}+\mathrm{H}\right]^{+}: 389.1614$, found: 389.1616<smiles>COc1ccc(/N=N/c2ccc(/C=N/NC(=O)c3ccncc3)cc2)cc1</smiles>

\section{0}

$N^{\prime}-((E)-4-((E)-(4-m e t h o x y p h e n y l)$ diazenyl)benzylidene)isonicotinohydrazide

Yield: $52.7 \mathrm{mg} \mathrm{(73 \% ).}$

Physical property: Orange sticky powder.

${ }^{1} \mathrm{H}$ NMR $\left(400 \mathrm{MHz}\right.$, DMSO- $\left.d_{6}\right) \delta 12.20(\mathrm{~s}, 1 \mathrm{H}), 8.84-8.76(\mathrm{~m}, 2 \mathrm{H}), 8.54(\mathrm{~s}, 1 \mathrm{H}), 7.97-7.88(\mathrm{~m}, 6 \mathrm{H}), 7.88-$ $7.82(\mathrm{~m}, 2 \mathrm{H}), 7.14(\mathrm{~d}, J=8.5,2 \mathrm{H}), 3.87(\mathrm{~s}, 3 \mathrm{H}) \mathrm{ppm}$

${ }^{13} \mathrm{C}$ NMR $\left(101 \mathrm{MHz}\right.$, DMSO- $\left.d_{6}\right) \delta 162.8,162.2,153.3,150.8,148.4,146.8,140.8,136.6,128.8,125.3$, 123.31, 122.03, 115.16, $56.16 \mathrm{ppm}$

HRMS (Orbitrap): Calcd for $\left[\mathrm{C}_{20} \mathrm{H}_{18} \mathrm{~N}_{5} \mathrm{O}_{2}{ }^{+}, \mathrm{M}+\mathrm{H}\right]^{+}: 360.1460$, found: 360.1457<smiles>COc1ccc(/N=N/c2ccc(/C=N/NC(=O)c3cccnc3)cc2)cc1</smiles>

\section{1}

$N^{\prime}-((E)-4-((E)-(4-m e t h o x y p h e n y l)$ diazenyl)benzylidene)nicotinohydrazide

Yield: $51 \mathrm{mg} \mathrm{(71 \% ).}$

Physical property: Orange sticky powder.

${ }^{1} \mathrm{H}$ NMR $\left(400 \mathrm{MHz}, \mathrm{DMSO}-\mathrm{d}_{6}\right) \delta 12.16(\mathrm{~s}, 1 \mathrm{H}), 9.09(\mathrm{~s}, 1 \mathrm{H}), 8.81-8.76(\mathrm{~m}, 1 \mathrm{H}), 8.52(\mathrm{~s}, 1 \mathrm{H}), 8.28(\mathrm{~d}, \mathrm{~J}=7.6$, $1 \mathrm{H}), 7.99-7.89(\mathrm{~m}, 6 \mathrm{H}), 7.62-7.55(\mathrm{~m}, 1 \mathrm{H}), 7.16(\mathrm{~d}, \mathrm{~J}=8.5,2 \mathrm{H}), 3.88(\mathrm{~s}, 3 \mathrm{H}) \mathrm{ppm}$

${ }^{13} \mathrm{C}$ NMR $\left(101 \mathrm{MHz}\right.$, DMSO- $\left.d_{6}\right) \delta 162.8,162.3,153.2,152.9,149.1,147.8,146.8,136.7,136.0,129.6,128.7$, 125.3, 124.1, 123.3, 115.2, $56.2 \mathrm{ppm}$

HRMS (Orbitrap): Calcd for $\left[\mathrm{C}_{20} \mathrm{H}_{18} \mathrm{~N}_{5} \mathrm{O}_{2}{ }^{+}, \mathrm{M}+\mathrm{H}\right]^{+}: 360.1460$, found: 360.1456<smiles>COc1ccc(/N=N/c2ccc(/C=N/NC(=O)CC#N)cc2)cc1</smiles>

22

2-cyano-N'-((E)-4-((E)-(4-methoxyphenyl)diazenyl)benzylidene)acetohydrazide

Yield: $57.8 \mathrm{mg} \mathrm{(94 \% ).}$ 
Physical property: Orange sticky powder.

${ }^{1} \mathrm{H}$ NMR $\left(400 \mathrm{MHz}, \mathrm{DMSO}-\mathrm{d}_{6}\right) \delta 11.96-11.82(\mathrm{~m}, 1 \mathrm{H}), 8.26-8.05(\mathrm{~m}, 1 \mathrm{H}), 7.96-7.87(\mathrm{~m}, 6 \mathrm{H}), 7.15(\mathrm{~d}$, $J=8.6,2 \mathrm{H}), 4.27(\mathrm{~s}, 2 \mathrm{H}), 3.89-3.82(\mathrm{~m}, 3 \mathrm{H}) \mathrm{ppm}$

${ }^{13} \mathrm{C}$ NMR $\left(101 \mathrm{MHz}\right.$, DMSO- $\left.d_{6}\right) \delta 165.5,162.8,159.6,153.2,153.1,147.2,146.8,143.9,136.4,136.3,128.8$, $128.5,128.3,125.2,123.7,123.2,123.2,120.3,116.5,116.2,115.3,115.2,56.2,25.4,24.9$ ppm

HRMS (Orbitrap): Calcd for $\left[\mathrm{C}_{17} \mathrm{H}_{16} \mathrm{~N}_{5} \mathrm{O}_{2}{ }^{+}, \mathrm{M}+\mathrm{H}\right]^{+}: 322.1304$, found: 322.1296

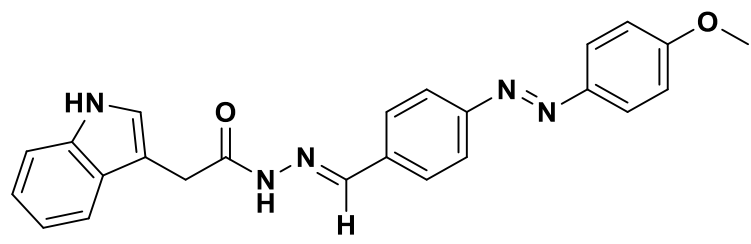

23

2-(1H-indol-3-yl)-N'-((E)-4-((E)-(4-methoxyphenyl)diazenyl)benzylidene)acetohydrazide

Yield: $68 \mathrm{mg}(83 \%)$

Physical property: Orange sticky powder.

${ }^{1} \mathrm{H}$ NMR $\left(400 \mathrm{MHz}\right.$, DMSO- $\left.d_{6}\right) \delta 11.70-11.40(\mathrm{~m}, 1 \mathrm{H}), 10.97-10.84(\mathrm{~m}, 1 \mathrm{H}), 8.37-8.04(\mathrm{~m}, 1 \mathrm{H}), 7.98-$ $7.83(\mathrm{~m}, 6 \mathrm{H}), 7.60(\mathrm{~d}, J=7.8,1 \mathrm{H}), 7.35(\mathrm{t}, J=8.0,1 \mathrm{H}), 7.30-7.22(\mathrm{~m}, 1 \mathrm{H}), 7.15(\mathrm{~d}, J=7.8,2 \mathrm{H}), 7.12-7.03(\mathrm{~m}$, $1 \mathrm{H}), 7.02-6.92(\mathrm{~m}, 1 \mathrm{H}), 4.10(\mathrm{~s}, 1 \mathrm{H}), 3.87(\mathrm{~s}, 3 \mathrm{H}), 3.67(\mathrm{~s}, 1 \mathrm{H}) \mathrm{ppm}$

${ }^{13} \mathrm{C}$ NMR $\left(101 \mathrm{MHz}\right.$, DMSO- $\left.d_{6}\right) \delta 173.3,167.7,162.7,153.0,152.8,146.8,145.5,142.0,138.0,136.6,136.5$, $128.4,128.2,127.9,125.2,124.4,123.3,123.2,121.5,121.4,119.2,119.1,118.9,118.8,115.2,111.8,111.8$, 108.5, 56.2, 32.2, $29.6 \mathrm{ppm}$

HRMS (Orbitrap): Calcd for $\left[\mathrm{C}_{24} \mathrm{H}_{22} \mathrm{~N}_{5} \mathrm{O}_{2}{ }^{+}, \mathrm{M}+\mathrm{H}\right]^{+}: 412.1773$, found: 412.1762

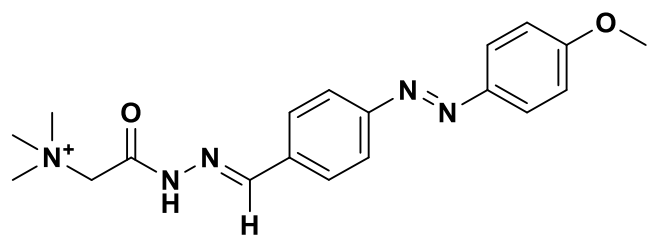

24

2-(2-((E)-4-((E)-(4-methoxyphenyl)diazenyl)benzylidene)hydrazineyl)-N,N,N-trimethyl-2-oxoethan-1aminium

Yield: $54 \mathrm{mg}(76 \%)$.

Physical property: Orange sticky powder.

${ }^{1} \mathrm{H}$ NMR $\left(400 \mathrm{MHz}\right.$, DMSO- $\left.d_{6}\right) \delta 12.87-12.19(\mathrm{~m}, 1 \mathrm{H}), 8.46-8.18(\mathrm{~m}, 1 \mathrm{H}), 7.95-7.89(\mathrm{~m}, 7 \mathrm{H}), 7.15(\mathrm{~d}$, $\mathrm{J}=8.2,3 \mathrm{H}), 4.85(\mathrm{~s}, 2 \mathrm{H}), 4.38(\mathrm{~s}, 1 \mathrm{H}), 3.88(\mathrm{~s}, 3 \mathrm{H}), 3.34(\mathrm{~s}, 9 \mathrm{H}) \mathrm{ppm}$

${ }^{13} \mathrm{C}$ NMR $\left(101 \mathrm{MHz}\right.$, DMSO- $\left.d_{6}\right) \delta 166.0,162.8,153.3,146.8,145.0,136.0,128.8,128.7,125.3,123.3,123.2$, $115.2,62.8,56.2,54.0,53.8 \mathrm{ppm}$

HRMS (Orbitrap): Calcd for $\left[\mathrm{C}_{19} \mathrm{H}_{24} \mathrm{~N}_{5} \mathrm{O}_{2}{ }^{+}, \mathrm{M}\right]^{+}: 354.1925$, found: 354.1924

\section{Experimental Procedure for Synthesizing 9}<smiles>O=[N+]([O-])c1ccc(CO)cc1</smiles>

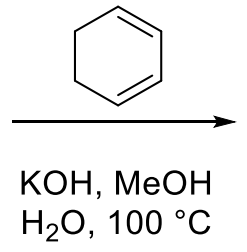<smiles>O=Cc1ccc(N2OC3C=CC2C3)cc1</smiles> 
A 2-5 mL Biotage microwave vial was charged with a stir bar, 4-nitrobenzyl alcohol 1 (76.5 mg, $0.5 \mathrm{mmol}, 1$ equiv), 1,3-cyclohexadiene (47.6 $\mu \mathrm{L}, 0.5 \mathrm{mmol} 1$ equiv), $10 \mathrm{M}$ potassium hydroxide (150 $\mu \mathrm{L}, 1.5 \mathrm{mmol}, 3$ equiv), methanol $(5 \mathrm{~mL})$ and water $(1.35 \mathrm{~mL})$. The vial was sealed with an aluminum crimp cap, and the reaction mixture was stirred and heated at $100{ }^{\circ} \mathrm{C}$ for $25 \mathrm{~h}$ in an oil bath. Methanol was removed by rotatory evaporation to afford a solid. The solid was transferred to a separatory funnel with water and extracted with dichloromethane $(3 \times 25 \mathrm{~mL})$. The organic layers were combined, dried with magnesium sulfate, and filtered. Dichloromethane was removed by rotatory evaporation. The solid was purified by flash column chromatography on silica gel (gradient elution: 19:1 $\rightarrow 7: 3$ hexanes:EtOAc).

Yield: $10 \mathrm{mg}(9 \%)$.

Physical property: Light yellow oil.

${ }^{1} \mathrm{H}$ NMR $\left(400 \mathrm{MHz}, \mathrm{CDCl}_{3}\right) \delta 9.85(\mathrm{~s}, 1 \mathrm{H}), 7.75(\mathrm{~d}, \mathrm{~J}=8.7,2 \mathrm{H}), 7.11(\mathrm{~d}, \mathrm{~J}=8.6,2 \mathrm{H}), 6.59(\mathrm{t}, \mathrm{J}=6.5,1 \mathrm{H}), 6.27(\mathrm{t}$, $J=6.9,1 \mathrm{H}), 4.83-4.78(\mathrm{~m}, 1 \mathrm{H}), 4.69-4.64(\mathrm{~m}, 1 \mathrm{H}), 2.36-2.24(\mathrm{~m}, 2 \mathrm{H}), 1.67-1.61(\mathrm{~m}, 1 \mathrm{H}), 1.50-1.39(\mathrm{~m}$, $1 \mathrm{H}) \mathrm{ppm}$

${ }^{13} \mathrm{C}$ NMR $\left(101 \mathrm{MHz}, \mathrm{CDCl}_{3}\right) \delta 191.0,157.6,131.6,130.9,130.3,130.1,116.2,70.0,55.4,23.9,21.0 \mathrm{ppm}$

HRMS (Orbitrap): Calcd for $\left[\mathrm{C}_{13} \mathrm{H}_{14} \mathrm{NO}_{2}{ }^{+}, \mathrm{M}+\mathrm{H}\right]^{+}:$: 216.1019 , found: 216.1017

\section{Experimental Procedures for Synthesizing 25}<smiles>COc1ccc(/N=N/c2ccc(C(=O)O)cc2)cc1</smiles>

\section{(E)-4-((4-methoxyphenyl)diazenyl)benzoic acid}

To a solution of p-anisidine $(0.3 \mathrm{~g}, 1.97 \mathrm{mmol})$ and 4-nitrosobenzoic acid ${ }^{1}(0.243 \mathrm{~g}, 1.97 \mathrm{mmol})$ in dichloromethane $\left(12 \mathrm{~mL}\right.$ ) was added acetic acid $(12 \mathrm{~mL})$. The reaction mixture was stirred under $\mathrm{N}_{2}$ at $35^{\circ} \mathrm{C}$ overnight. Upon cooling to $0{ }^{\circ} \mathrm{C}$, a precipitate formed, which was collected via vacuum filtration and used without further purification. Spectral characteristics are in accordance with the previously published literature. ${ }^{2}$

Yield: $377 \mathrm{mg}(75 \%)$

Physical property: Orange solid

${ }^{1} \mathrm{H}$ NMR $\left(400 \mathrm{MHz}\right.$, DMSO-d $\left.{ }_{6}\right) \delta 13.2(\mathrm{br} \mathrm{s}, 1 \mathrm{H}), 8.10(\mathrm{~d}, J=5.6,2 \mathrm{H}), 7.94(\mathrm{~d}, J=6.4,2 \mathrm{H}), 7.90(\mathrm{~d}, J=5.6$, $2 \mathrm{H}), 7.14(\mathrm{~d}, J=6.4,2 \mathrm{H}), 3.88(\mathrm{~s}, 3 \mathrm{H}) \mathrm{ppm}$

${ }^{13}$ C NMR $(125 \mathrm{MHz}$, DMSO-d 6 ) $\delta 167.6,163.4,155.3,147.1,133.0,131.4,125.8,123.1,115.6,56.6$ ppm<smiles>COC(=O)c1ccc(/N=N/c2ccc(OC)cc2)cc1</smiles>

\section{methyl (E)-4-((4-methoxyphenyl)diazenyl)benzoate}

To a solution of $(E)-4-((4-m e t h o x y p h e n y l)$ diazenyl)benzoic acid $(0.355 \mathrm{~g}, 1.39 \mathrm{mmol})$ in methanol $(14 \mathrm{~mL})$ was added concentrated hydrochloric acid $(0.35 \mathrm{~mL})$. The mixture was refluxed for $18 \mathrm{~h}$, cooled to room temperature, and concentrated in vacuo. The resulting residue was diluted with dichloromethane $(50 \mathrm{~mL})$ and $\mathrm{H}_{2} \mathrm{O}(50 \mathrm{~mL})$. The aqueous layer was neutralized with saturated aqueous $\mathrm{NaHCO}_{3}$ and extracted with 
dichloromethane $(3 \times 50 \mathrm{~mL})$, dried over $\mathrm{Na}_{2} \mathrm{SO}_{4}$, concentrated in vacuo, and used without further purification Spectral characteristics are in accordance with the previously published literature. ${ }^{3}$

Yield: $389.1 \mathrm{mg}(>99 \%)$

Physical property: Orange solid

${ }^{1} \mathrm{H}$ NMR $\left(400 \mathrm{MHz}, \mathrm{CDCl}_{3}\right) \delta 8.20(\mathrm{~d}, J=8.8 \mathrm{~Hz}, 2 \mathrm{H}), 7.98(\mathrm{~d}, J=8.4 \mathrm{~Hz}, 2 \mathrm{H}), 7.93(\mathrm{~d}, J=8.4 \mathrm{~Hz}, 2 \mathrm{H}), 7.06(\mathrm{~d}$, $J=8.8 \mathrm{~Hz}, 2 \mathrm{H}), 3.98(\mathrm{~s}, 3 \mathrm{H}), 3.94(\mathrm{~s}, 3 \mathrm{H})$

${ }^{13} \mathrm{C}$ NMR $\left(125 \mathrm{MHz}, \mathrm{CDCl}_{3}\right) \delta 166.64,162.65,155.34,146.99,131.13,130.58$ 125.18, 122.35, 114.32, 55.62, $52.28 \mathrm{ppm}$<smiles>COc1ccc(/N=N/c2ccc(C(=O)NN)cc2)cc1</smiles>

\section{(E)-4-((4-methoxyphenyl)diazenyl)benzohydrazide}

A 2-5 mL biotage microwave vial was charged with ethanol $(1.75 \mathrm{~mL})$, methyl $(E)-4-((4-$ methoxyphenyl)diazenyl)benzoate $(0.142 \mathrm{~g}, 0.53 \mathrm{mmol})$, and hydrazine monohydrate $(0.064 \mathrm{~mL}, 1.32 \mathrm{mmol})$. The vial was sealed with an aluminum crimp cap and microwaved at $150^{\circ} \mathrm{C}$ for $5 \mathrm{~h}$. The reaction was cooled to $0^{\circ} \mathrm{C}$ and the precipitate was collected via vacuum filtration and washed with cold ethanol.

Yield: $69.0 \mathrm{mg} \mathrm{(49 \% )}$

Physical property: Orange solid

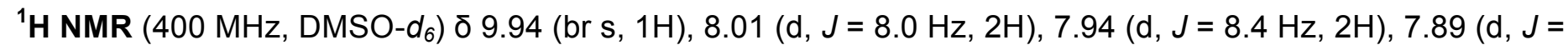
$8.8 \mathrm{~Hz}, 2 \mathrm{H}), 7.17(\mathrm{~d}, J=\mathrm{Hz}, 2 \mathrm{H}), 4.06$ (br s, 2H), 3.89 (s, 3H) ppm

${ }^{13}$ C NMR $(125 \mathrm{MHz}$, DMSO-d 6 ) $\delta 165.54,162.85,153.81,146.64,135.24,128.64,125.32,122.51,115.15$, $56.15 \mathrm{ppm}$<smiles>COc1ccc(/N=N/c2ccc(C(=O)N/N=C/c3ccccc3)cc2)cc1</smiles>

\section{N'-((E)-4-((E)-(4-methoxyphenyl)diazenyl)benzylidene)benzohydrazide}

A 2-5 mL Biotage microwave vial was charged with a stir bar, ethanol $(2.4 \mathrm{~mL}),(E)-4-((4-$ methoxyphenyl)diazenyl)benzohydrazide $(0.065 \mathrm{~g}, 0.24 \mathrm{mmol})$, benzaldehyde $(0.025 \mathrm{~mL}, 0.24 \mathrm{mmol})$ and a catalytic amount of trifluoroacetic acid. The vial was sealed with an aluminum crimp cap. The reaction mixture was stirred and heated at $80^{\circ} \mathrm{C}$ overnight. The reaction was cooled to $0{ }^{\circ} \mathrm{C}$ and the orange precipitate collected via vacuum filtration and washed with cold ethanol.

Yield: $42 \mathrm{mg}(51 \%)$

\section{Physical property: Orange solid}

${ }^{1} \mathrm{H}$ NMR $\left(400 \mathrm{MHz}\right.$, DMSO- $\left.d_{6}\right) \delta 12.00(\mathrm{~s}, 1 \mathrm{H}), 8.50(\mathrm{~s}, 1 \mathrm{H}), 8.12(\mathrm{~d}, J=8.2 \mathrm{~Hz}, 2 \mathrm{H}), 7.93-7.96(\mathrm{~m}, 4 \mathrm{H}), 7.76$ $(\mathrm{d}, J=6.8 \mathrm{~Hz}, 2 \mathrm{H}), 7.45-7.48(\mathrm{~m}, 3 \mathrm{H}), 7.16(\mathrm{~d}, J=8.8 \mathrm{~Hz}, 2 \mathrm{H}), 3.88(\mathrm{~s}, 3 \mathrm{H}) \mathrm{ppm}$ 
${ }^{13} \mathrm{C}$ NMR $\left(101 \mathrm{MHz}\right.$, DMSO-d $\left.\mathrm{d}_{6}\right) \delta 163.00,162.95,153.25,148.65,146.71,135.28,134.74,130.67,129.43$, $129.35,127.65,125.44,122.67,115.22,56.21 \mathrm{ppm}$

HRMS (Orbitrap): Calcd for $\left[\mathrm{C}_{21} \mathrm{H}_{19} \mathrm{~N}_{4} \mathrm{O}_{2}{ }^{+}, \mathrm{M}+\mathrm{H}\right]^{+}: 359.1508$, found: 359.1503

\section{Constant Illumination NMR Experiments}

Compounds were dissolved in either DMSO- $d_{6}$ or $\mathrm{D}_{2} \mathrm{O}$ with the sample volume and concentration being 450 $\mu \mathrm{L}$ and $100 \mu \mathrm{M}$, respectively. Samples were illuminated using a Mightex WheeLED light source equipped with a fiber optic cable fed directly into the NMR tube. The tip of the fiber optic cable was placed just above the solvent, and the sample was manually lowered into an $800 \mathrm{MHz} N M R$ instrument. Ratios of isomers were measured at $365 \mathrm{~nm}, 405 \mathrm{~nm}, 470 \mathrm{~nm}, 530 \mathrm{~nm}, 590 \mathrm{~nm}, 625 \mathrm{~nm}, 656 \mathrm{~nm}, 740 \mathrm{~nm}$, and $850 \mathrm{~nm}$ in order of increasing wavelength. Data was collected after 30 seconds of switching the wavelength. The power output for the LEDs ranged from $1-18 \mathrm{~mW} / \mathrm{cm}^{2}$ depending on the specific wavelength. Half-lives for compounds 13 and 24 were determined by $1 \mathrm{H}$ NMR. A $100 \mu \mathrm{M}$ sample was illuminated with $365 \mathrm{~nm}$ light for 5 minutes to convert the compound to predominantly the $Z$ isomer. The light was then turned off and the sample was kept in the dark. The sample was monitored for up to 100 minutes for conversion to the $E$ isomer. The data, concentration of the $Z$ isomer versus time, was plotted in GraphPad Prism 8 with an exponential decay best fit line constrained to an asymptote at the $\mathrm{x}$-axis to reflect the dark state $1 \mathrm{H}$ NMR data presented which is comprised of solely the more thermodynamically stable $E$ isomer. Half-lives were extrapolated from the equation of the best fit line.

\section{UV-Vis Characterization for Compounds 8, 13, and 25}

UV-Vis spectra were recorded on a Nanodrop 2000c spectrophotometer. $3 \mathrm{~mL}$ of $50 \mu \mathrm{M}$ (DMSO) samples in a quartz cuvette were illuminated by a Mightex WheeLED light source equipped with a fiber optic cable for 10 min at each successive wavelength at ambient temperature with stirring that was ceased momentarily while recording spectra.

\section{Photoswitchable Surfactant Experiments}

A solution of aqueous photoswitchable surfactant $24(2.5 \mu \mathrm{L}, 0.05 \mathrm{M})$ or aqueous cetrimonium bromide (2.5 $\mu \mathrm{L}, 2.5 \%(\mathrm{w} / \mathrm{w}))$ was added to benzene $(7.5 \mu \mathrm{L})$ and vortexed for ten seconds. The biphasic solutions were each placed on separate microscope slides, covered with coverslips, and sealed with vinyl polysiloxane (Exaflex Injection Type Impression Material, GC America) to prevent evaporation. The slides were then imaged under brightfield illumination with a Leica inverted epifluorescence microscope (DM IL) using a 10x objective (Leica, HC PL Fluotar, 0.30 NA). Photoisomerization was induced using an Excelitas Technologies X-Cite 120 LED mini with the excitation bandpass filters AT350/50x or ET560/40x (Chroma). Filters were switched manually. Fluorescence images were acquired with the excitation, dichroic, and emission filters ET470/40x, ET525/50m, and T495LPXR, respectively (Chroma). Images and movies were recorded at 100 ms exposure with a Retiga R1 CCD (Teledyne QImaging) and Ocular software. Analysis was performed in ImageJ Fiji (version 1.520). 


\section{Crystallography Information for 7, 8, and 19}

Crystallographic data were collected using a Bruker SMART APEX II diffractometer equipped with monochromatic Mo Ka $(\lambda=0.71073 \AA)$ radiation or a Bruker D8 VENTURE Kappa Duo PHOTON 100 diffractometer with Cu-Ka radiation $(\lambda=1.54178 \AA$ ). The diffraction data were reduced by SAINT software and absorption corrections were applied with SADABS software. The crystal structures were solved with direct methods by using SHELXTL package and refined with SHELXL 2013 program. The hydrogen atoms were refined with a riding model.

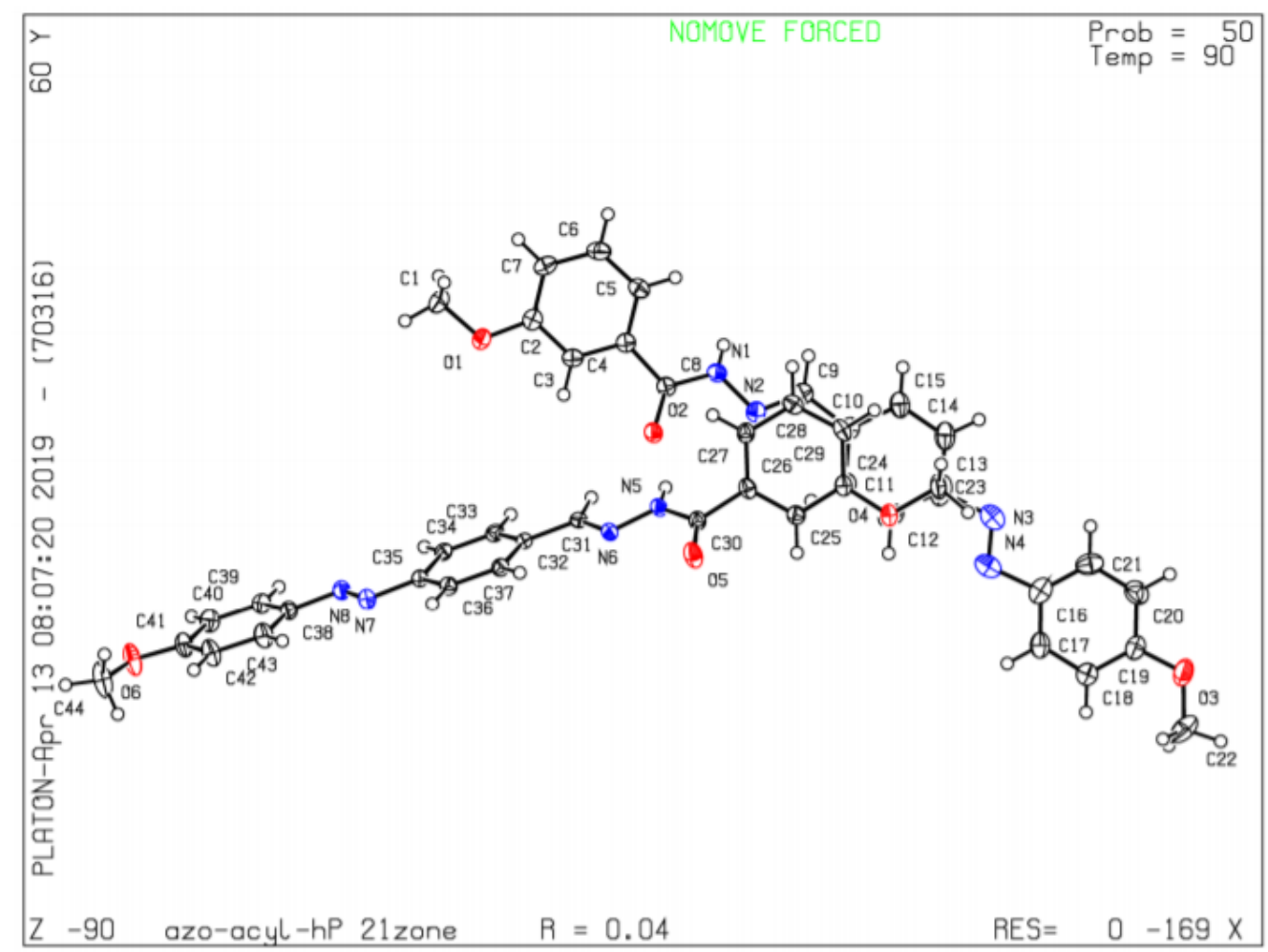



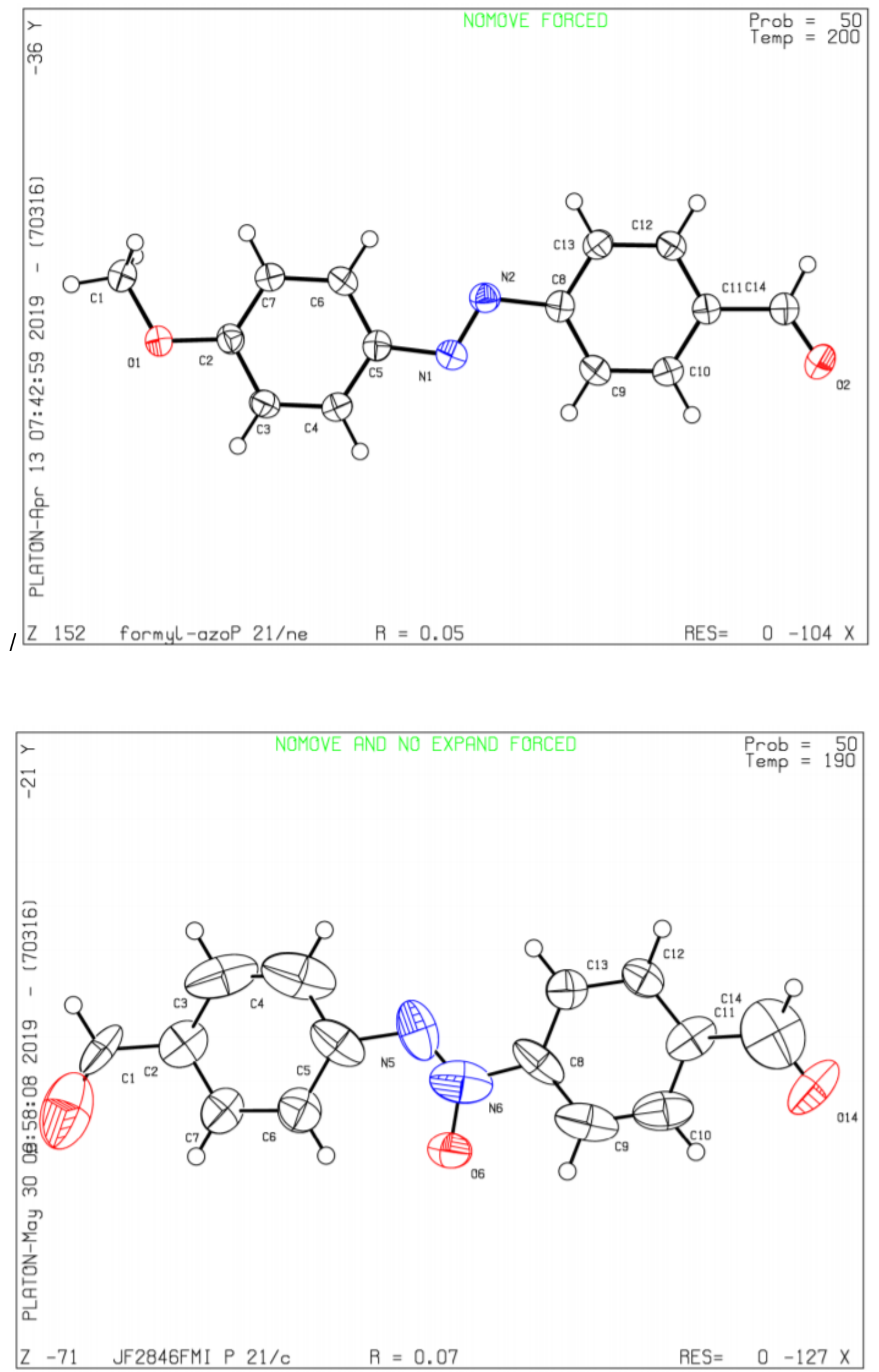
Table S2. Crystallographic parameters

\begin{tabular}{|c|c|c|c|}
\hline & 8 & 19 & 7 \\
\hline Empirical formula & $\mathrm{C}_{14} \mathrm{H}_{12} \mathrm{~N}_{2} \mathrm{O}_{2}$ & $\mathrm{C}_{22} \mathrm{H}_{20} \mathrm{~N}_{4} \mathrm{O}_{3}$ & $\mathrm{C}_{14} \mathrm{H}_{10} \mathrm{~N}_{2} \mathrm{O}_{3}$ \\
\hline Formula weight & 240.26 & 388.42 & 254.24 \\
\hline $\mathrm{T}(\mathrm{K})$ & $200(2)$ & $90(2)$ & $190(2)$ \\
\hline Crystal system & Monoclinic & Monoclinic & Monoclinic \\
\hline Space group & $P 2_{1} / n$ & $P 2_{1}$ & $P 2_{1} / c$ \\
\hline Color/Shape & Orange/Plate & Orange/Block & Yellow/Plate \\
\hline$a(\AA)$ & $15.2381(5)$ & $7.8457(4)$ & $6.2109(2)$ \\
\hline$b(\AA)$ & $4.59000(10)$ & $28.2311(15)$ & $4.58210(10)$ \\
\hline$c(\AA)$ & $16.7961(5)$ & $9.0217(5)$ & $20.6105(8)$ \\
\hline$\alpha(\mathrm{deg})$ & 90 & 90 & 90 \\
\hline$\beta$ (deg) & $95.1750(10)$ & $101.9340(10)$ & $96.380(2)$ \\
\hline$V(\mathrm{deg})$ & 90 & 90 & 90 \\
\hline$V\left(\AA^{3}\right)$ & 1169.98(6) & $1955.05(18)$ & $582.92(3)$ \\
\hline Z & 4 & 4 & 2 \\
\hline$\rho_{\text {calcd }}\left(\mathrm{g} \mathrm{cm}^{-3}\right)$ & 1.364 & 1.320 & 1.448 \\
\hline$\mu\left(\mathrm{mm}^{-1}\right)$ & 0.082 & 0.090 & 0.866 \\
\hline Crystal size $(\mathrm{mm})$ & $0.28 \times 0.27 \times 0.08$ & $0.85 \times 0.47 \times 0.34$ & $0.29 \times 0.24 \times 0.04$ \\
\hline$\theta$ range $(\mathrm{deg})$ & 3.75 to 72.27 & 2.31 to 27.60 & 4.32 to 72.31 \\
\hline $\begin{array}{l}\text { Data / restraints / } \\
\text { parameters }\end{array}$ & $2313 / 0 / 164$ & $9046 / 1 / 535$ & 1148 / 14 / 149 \\
\hline GOF on $F^{2}$ & 1.097 & 1.040 & 1.073 \\
\hline $\begin{array}{l}\mathrm{R} 1 / \mathrm{wR} 2 \\
{[\mathrm{I}>2 \operatorname{sigma}(\mathrm{I})]}\end{array}$ & $0.0450 / 0.1113$ & $0.0400 / 0.1046$ & $0.0744 / 0.1893$ \\
\hline R1/wR2 (all data) & $0.0560 / 0.1189$ & $0.0409 / 0.1054$ & $0.0885 / 0.2008$ \\
\hline \multicolumn{4}{|c|}{ Extinction coefficient } \\
\hline $\begin{array}{l}\text { Largest diff. } \\
\text { peak/hole }\left(e \cdot \AA^{-3}\right)\end{array}$ & $0.250 /-0.349$ & $0.813 /-0.285$ & $0.239 /-0.184$ \\
\hline
\end{tabular}




\section{Structural Determination and Refinement for 7:}

All crystallographic calculations were performed on an Intel Xeon E5-1620v2 at 3.70GHz an eight core processor and 16GB of extended memory. Data collected were corrected for Lorentz and polarization effects with Saint ${ }^{4}$ and absorption using Blessing's method and merged as incorporated with the program Sadabs. ${ }^{5,6}$ The SHELXTL ${ }^{7}$ program package was implemented to determine the probable space group and set up the initial files. System symmetry, systematic absences and intensity statistics indicated the non-centrosymmetric monoclinic space group $\mathrm{P} 2{ }_{1} / \mathrm{c}$ (no. 14). The structure was determined by direct methods with the non-hydrogen atoms being located directly for half of the molecule that sits on a special position using the program $\mathrm{XT}$. $^{8} \mathrm{The}$ structure was refined with $\mathrm{XL} .{ }^{9}$ The data collected were merged for least squares refinement to 1148 unique data $[R($ int $)=0.0214]$. The large thermal motion and large number of residual peaks indicated that the structure suffered from whole molecule disorder so the molecule was initially 'grown', then a series of restraints implemented to idealize the phenyl rings and various other parts of the molecule. The molecule was now input at half occupancy and allowed to refine. All of the residual intensity was absorbed by the molecule and its various modes of vibration. All non-hydrogen atoms were refined anisotropically. Hydrogen atoms were idealized throughout the final refinement. The suboptimal thermal parameters are a result of the whole molecule disorder with the two orientations attempting to model all of the electron density present in its disordered form. The data collected were extremely weak at higher angles that resulted in larger than desired final residuals. The final structure was refined to convergence with $R(F)=8.85 \%, w R\left(F^{2}\right)=20.08 \%, G O F=1.073$ for all 1148 unique reflections $\left[R(F)=7.44, w R\left(F^{2}\right)=18.93 \%\right.$ for those 915 data with Fo $\left.>4 \sigma(F o)\right]$. The final difference-Fourier map was featureless indicating that the structure is both correct and complete.

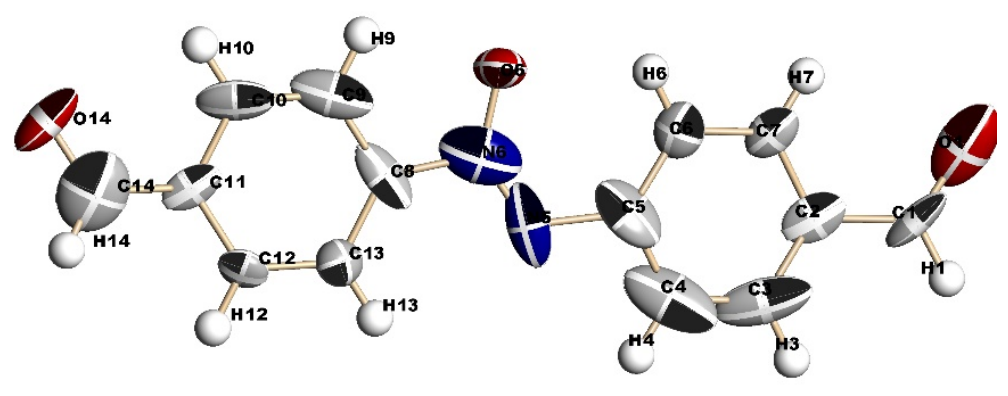

\section{Computational Details}

Density Functional Theory (DFT) calculations were performed using Gaussian 09 Revision D.01, and surfaces were rendered using GaussView 6. ${ }^{10}$ Ground state structures were first optimized at B3LYP/3-21G PCM(DMSO). ${ }^{11}$ cam-B3LYP-GD3BJ/6-311+G(d,p) PCM(DMSO) was then utilized for further geometry refinement and for time-dependent DFT (TD-DFT) calculations in the excited state. The coulomb-attenuating method $^{12}$ was chosen due to the degree of conjugation in our systems. Empirical dispersion ${ }^{13}$ and damping were included as these effects are likely to be important in Z-isomers of the switchable moieties given the steric crowding that prevents planarity of the system. PCM solvation corrections were included in both the ground and excited states using the standard linear response solvation method within Gaussian. Overall, this level of theory accurately reproduced experimentally observed spectra for (E)-1,2-diphenyldiazene and (E)-N'benzylidenebenzhydrazide ${ }^{14}$ (not presented) as an internal quality check. Excited state densities were calculated for the $\pi$ to $\pi^{*}$ transition for E-azo isomers and for the $n$ to $\pi^{*}$ transition for Z-azo isomers. Differential electron densities between the excited and ground states were then mapped in GaussView to better visualize the localization of electron density in the excited state. 


\section{Supplemental References}

1. Priewisch, B, Rück-Braun, K. Efficient preparation of nitrosoarenes for the synthesis of azobenzenes. J. Org. Chem. 2005, 70, 2350-2352.

2. Kreger, K., Wolfer, P., Audorff, H., Kador, L., Stingelin-Stutzmann, N., Smith, P., Schmidt, H. Stable holographic gratings with small-molecular trisazobenzene derivatives. J. Am. Chem. Soc. 2010, 132, 509516.

3. Davey, M. H., Lee, V. Y., Miller, R. D., Marks, T. J. Synthesis of aryl nitroso derivatives by tert-butyl hypochlorite oxidation in homogeneous media. Intermediates for the preparation of high-hyperpolarizability chromaphore skeletons. J. Org. Chem. 1999, 64, 4976-4979.

4. Bruker (2017) APEX3 (Version 2017.3) and (2016) SAINT (Version 8.37a). Bruker AXS Inc., Madison, Wisconsin, USA.

5. Blessing, R. H., An Empirical Correction for Absorption Anisotropy. Acta Cryst., 1995, A51, 33-38.

6. Sheldrick, G.M., SADABS (2016) Version 2016/2, 'Siemens Area Detector Absorption Correction' Universität Göttingen: Göttingen, Germany.

7. Sheldrick, G.M., (2002). SHELXTL. Version 6.1. Bruker AXS Inc., Madison, Wisconsin, USA.

8. Sheldrick, G. M., (2014) SHELXT, Universität Göttingen: Göttingen, Germany. Structure determination program. Private communication.

9. Sheldrick, G. M., (2017). SHELXL2017/1. Universität Göttingen: Göttingen, Germany.

10. M. J. Frisch, G. W. T., H. B. Schlegel, G. E. Scuseria, M. A. Robb, J. R. Cheeseman, G. Scalmani, V. Barone, G. A. Petersson, H. Nakatsuji, X. Li, M. Caricato, A. Marenich, J. Bloino, B. G. Janesko, R. Gomperts, B. Mennucci, H. P. Hratchian, J. V. Ortiz, A. F. Izmaylov, J. L. Sonnenberg, D. Williams-Young, F. Ding, F. Lipparini, F. Egidi, J. Goings, B. Peng, A. Petrone, T. Henderson, D. Ranasinghe, V. G. Zakrzewski, J. Gao, N. Rega, G. Zheng, W. Liang, M. Hada, M. Ehara, K. Toyota, R. Fukuda, J. Hasegawa, M. Ishida, T. Nakajima, Y. Honda, O. Kitao, H. Nakai, T. Vreven, K. Throssell, J. A. Montgomery, Jr., J. E. Peralta, F. Ogliaro, M. Bearpark, J. J. Heyd, E. Brothers, K. N. Kudin, V. N. Staroverov, T. Keith, R. Kobayashi, J. Normand, K. Raghavachari, A. Rendell, J. C. Burant, S. S. Iyengar, J. Tomasi, M. Cossi, J. M. Millam, M. Klene, C. Adamo, R. Cammi, J. W. Ochterski, R. L. Martin, K. Morokuma, O. Farkas, J. B. Foresman, and D. J. Fox Gaussian 09 Revision D.01, Gaussian, Inc.: Wallingford CT, 2016.

11. Scalmani, G.; Frisch, M. J., Continuous surface charge polarizable continuum models of solvation. I. General formalism. J. Chem. Phys. 2010, 132, 114110.

12. Yanai, T.; Tew, D. P.; Handy, N. C., A new hybrid exchange-correlation functional using the Coulombattenuating method (CAM-B3LYP). Chem. Phys. Lett. 2004, 393, 51-57.

13. Wagner, J. P.; Schreiner, P. R., London dispersion in molecular chemistry-reconsidering steric effects. Angew. Chem. Int. Ed. 2015, 54, 12274-12296.

14. van Dijken, D. J.; Kovaříček, P.; Ihrig, S. P.; Hecht, S., Acylhydrazones as Widely Tunable Photoswitches. J. Am. Chem. Soc. 2015, 137, 14982-14991. 


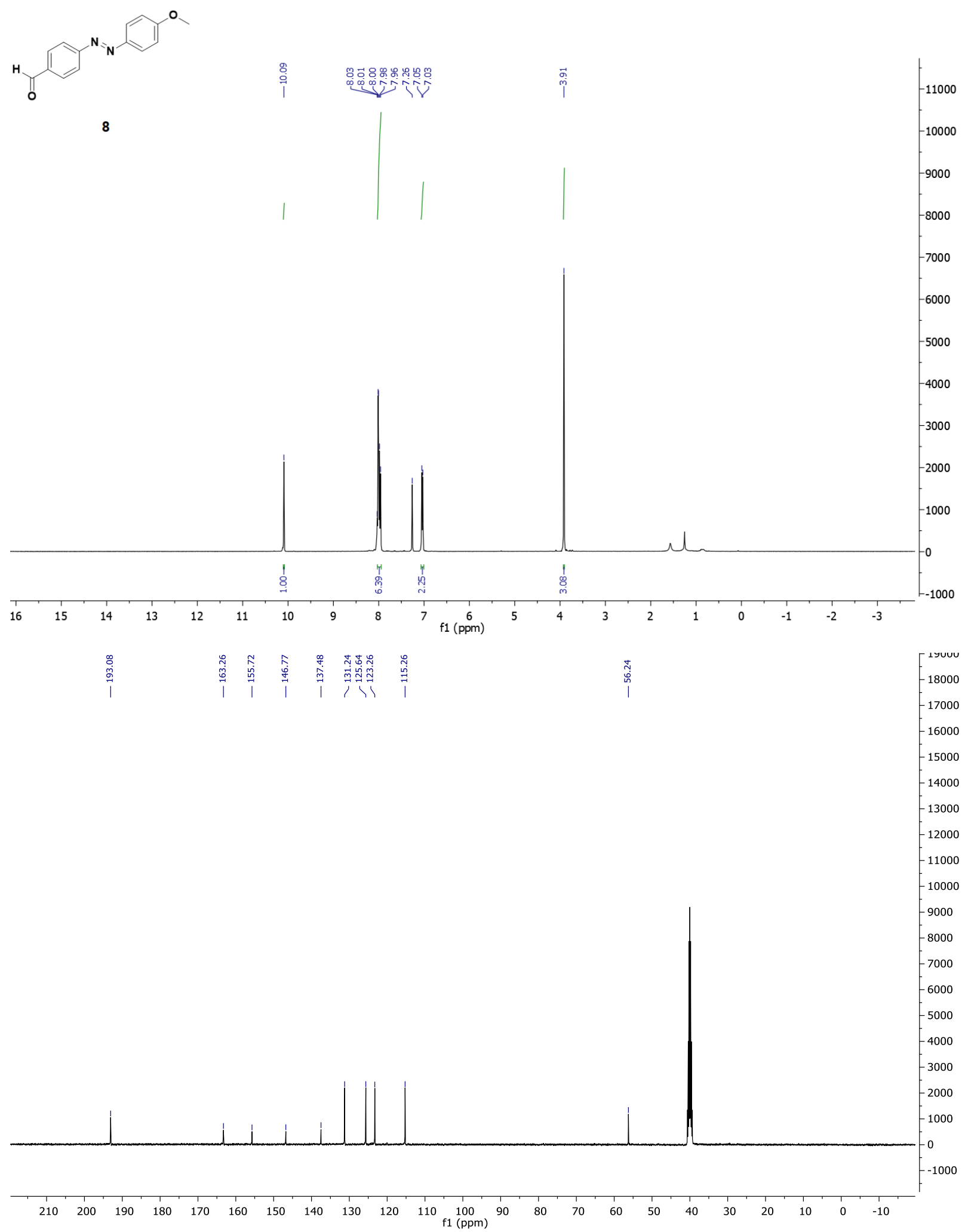




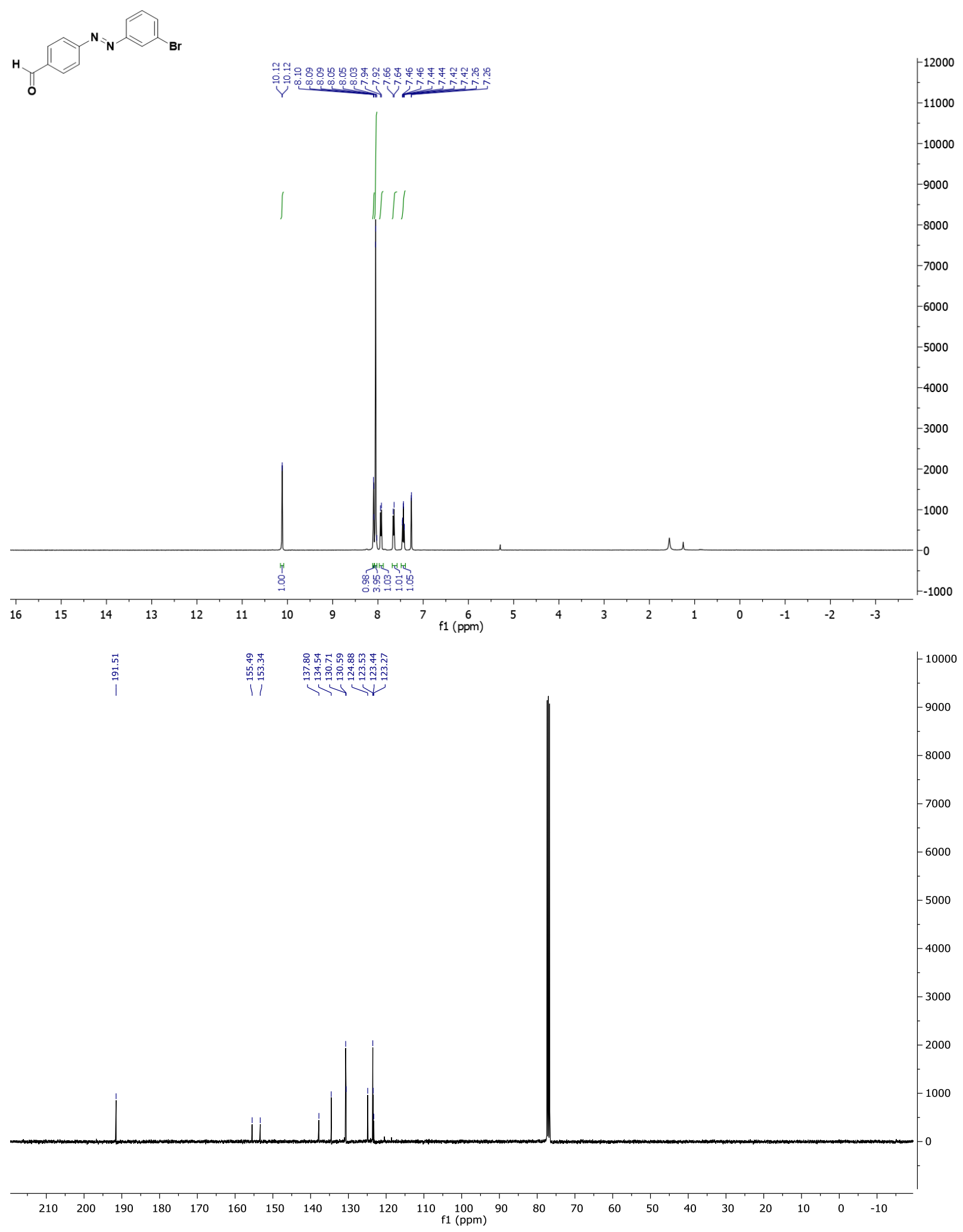




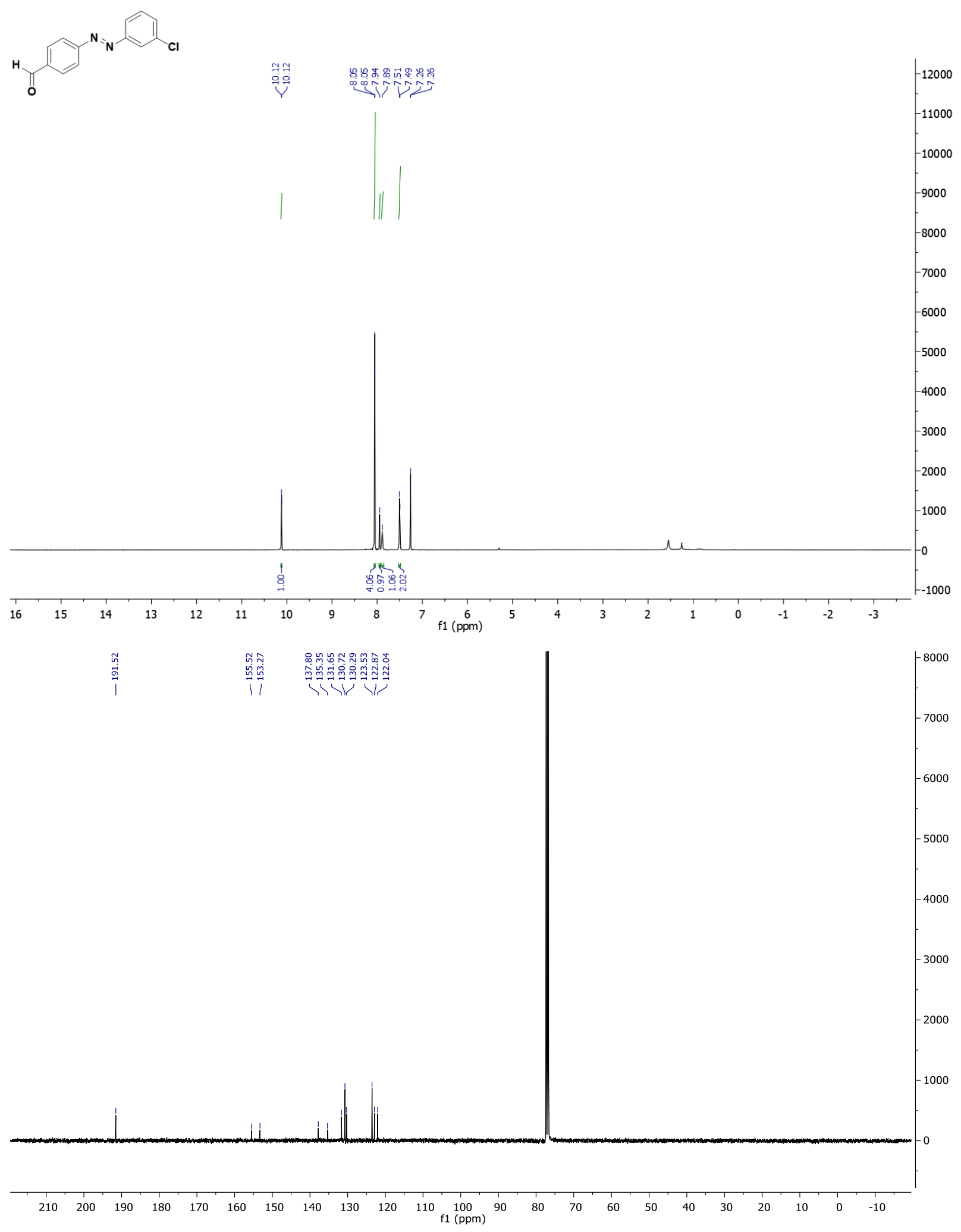




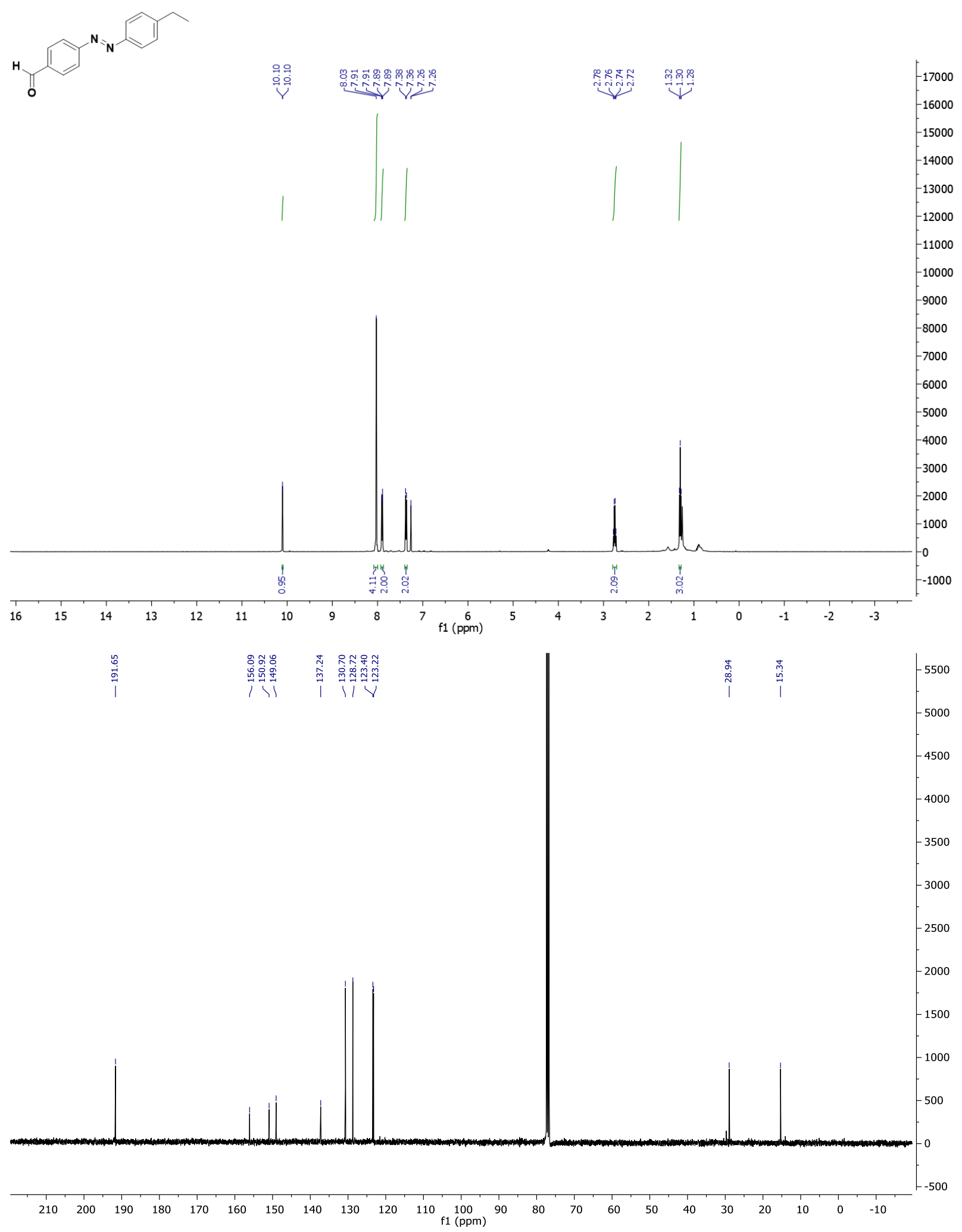




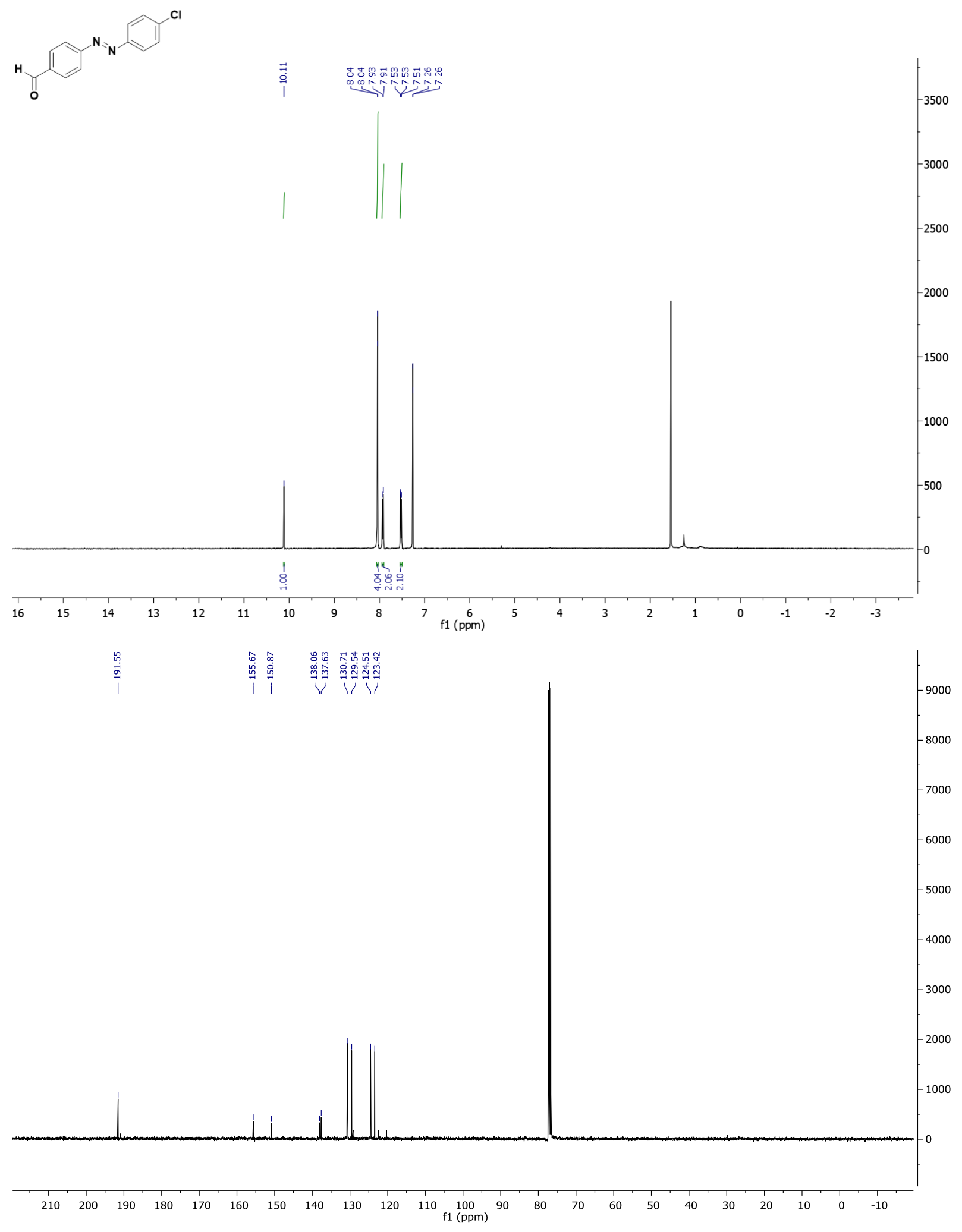




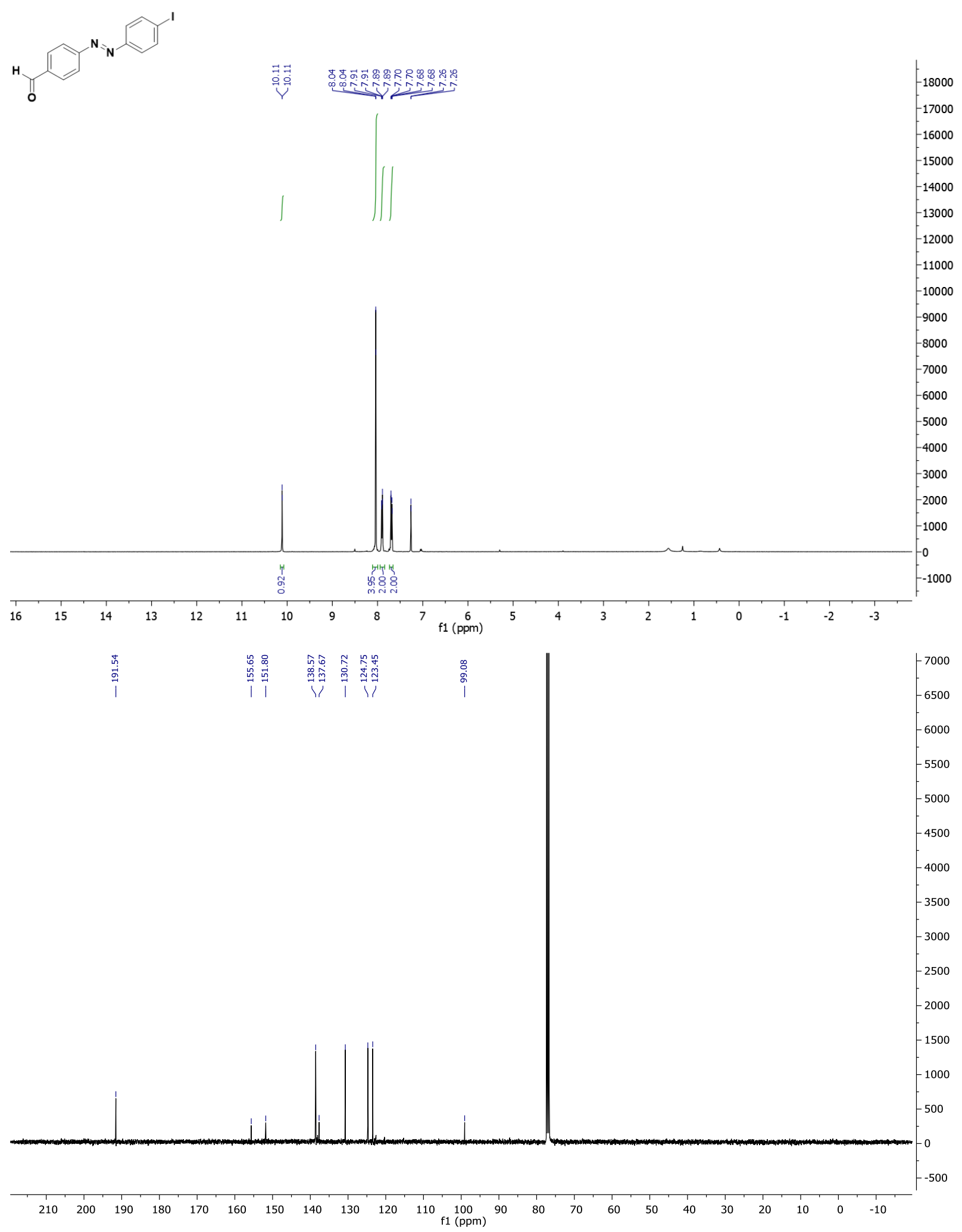




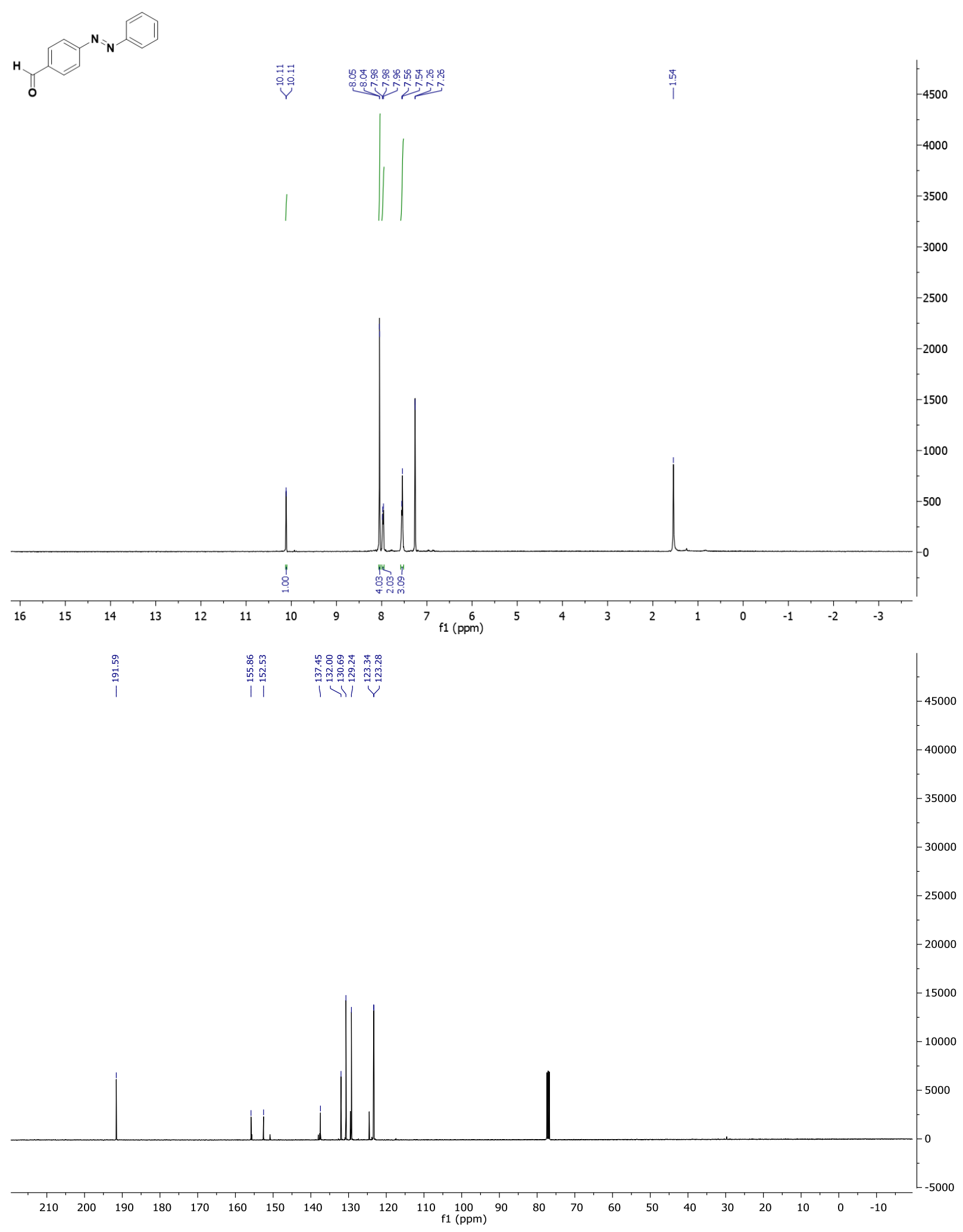




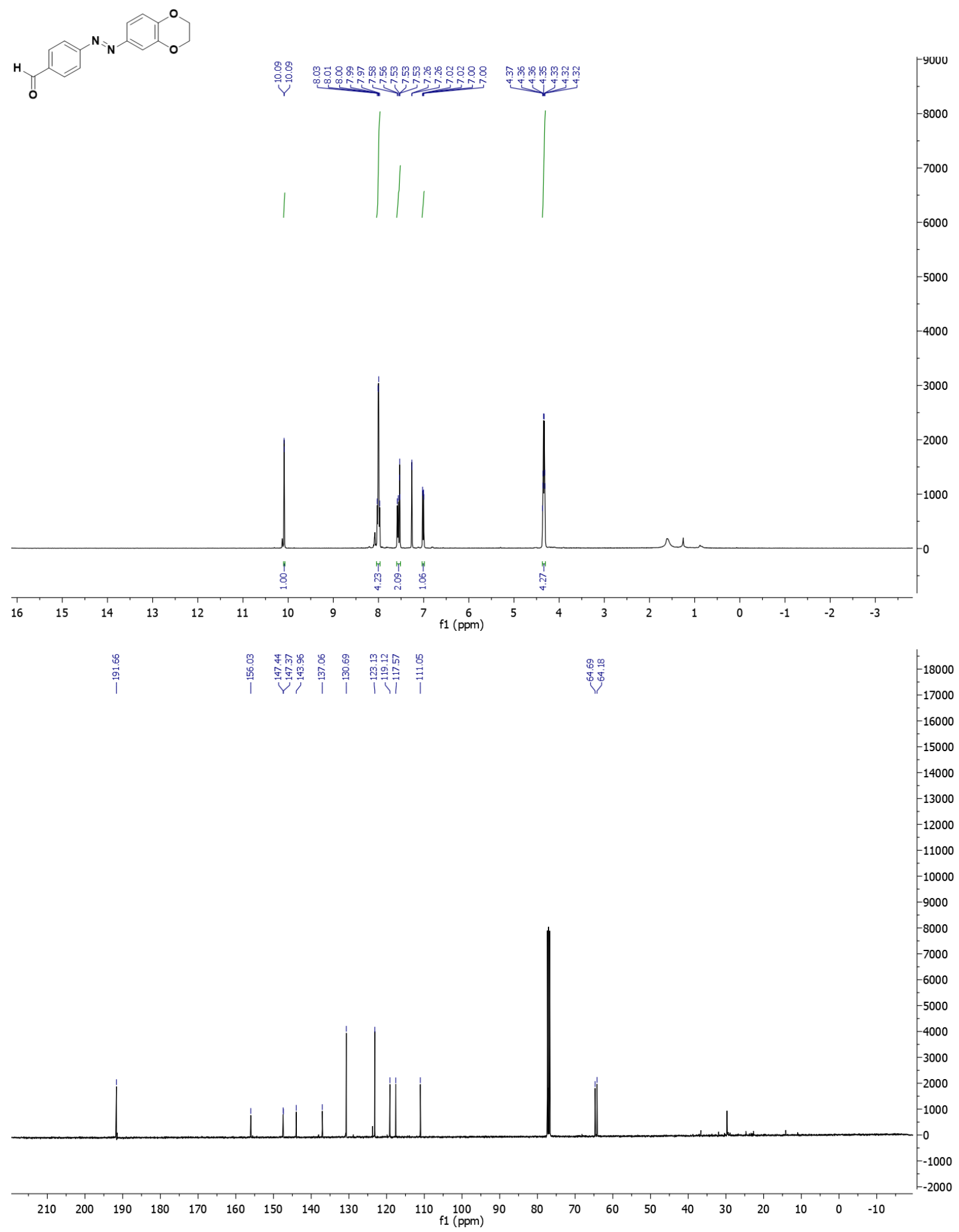




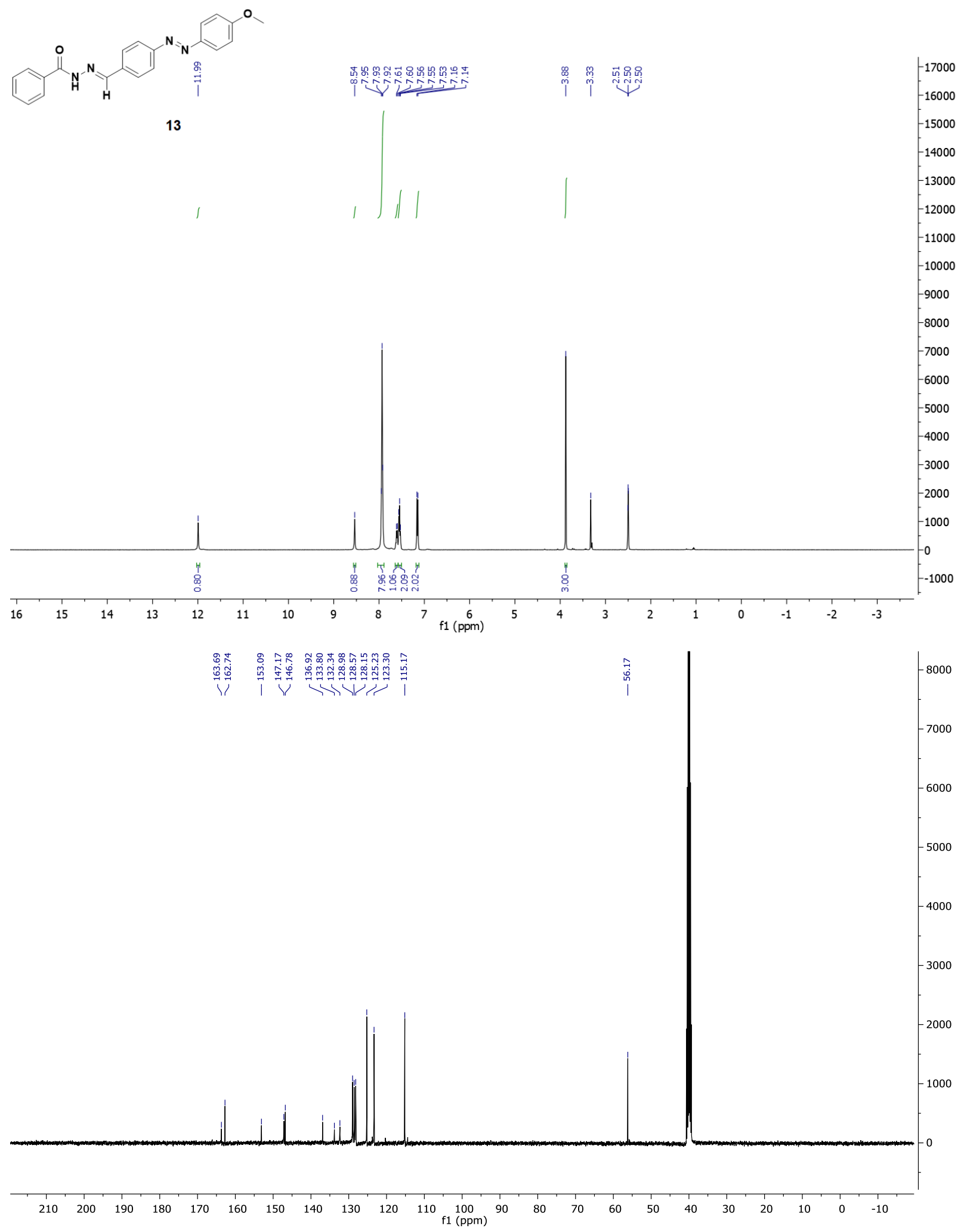




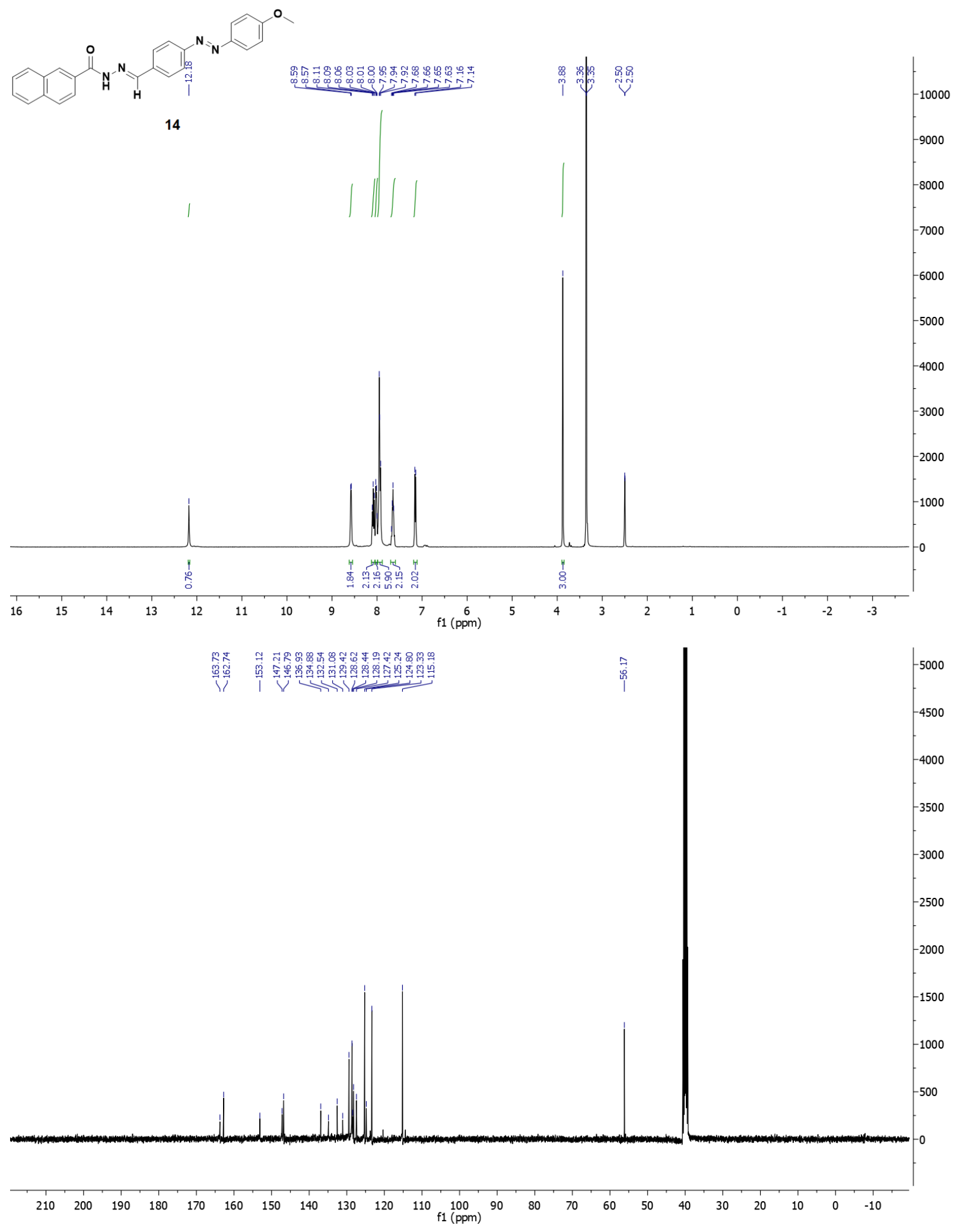




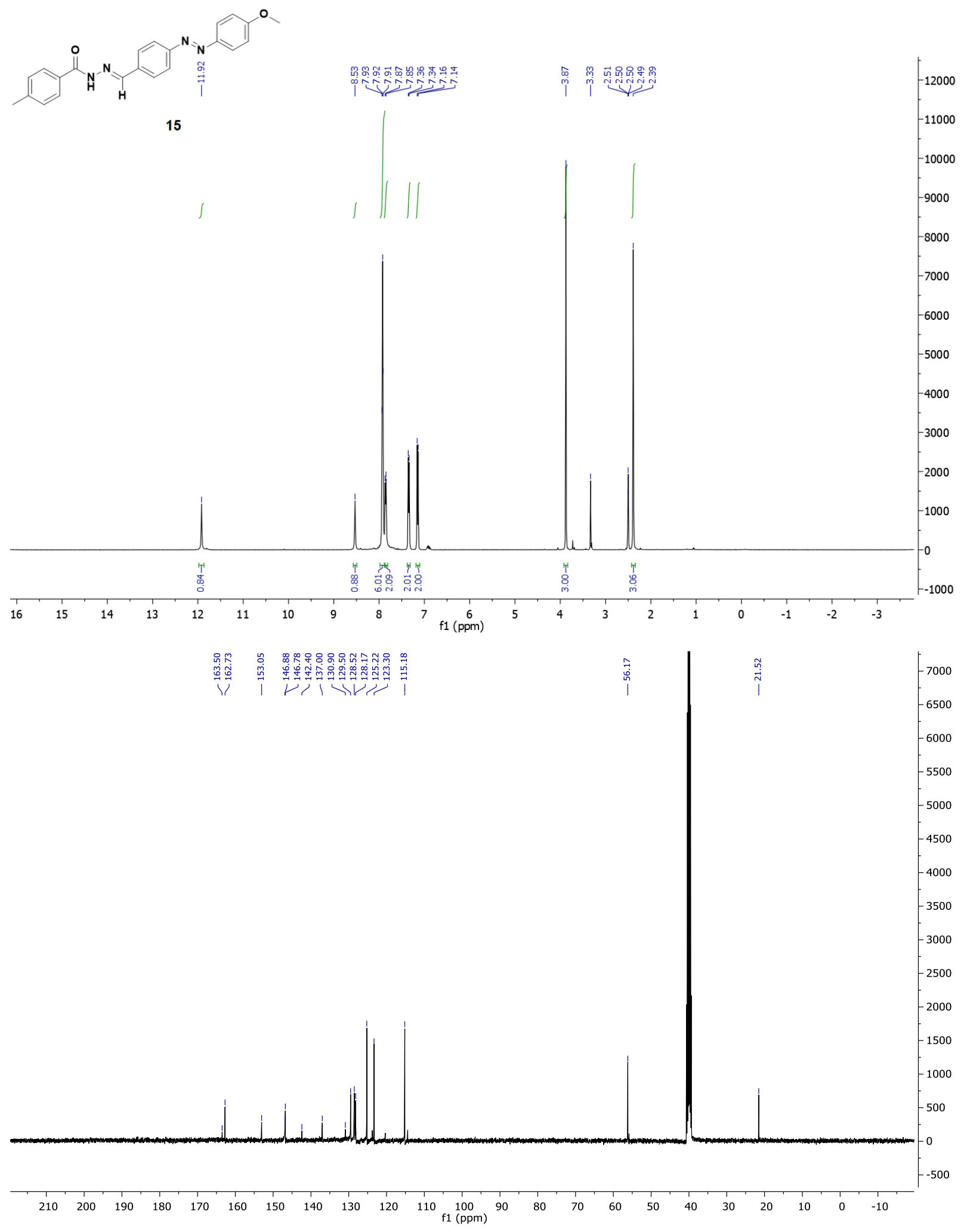




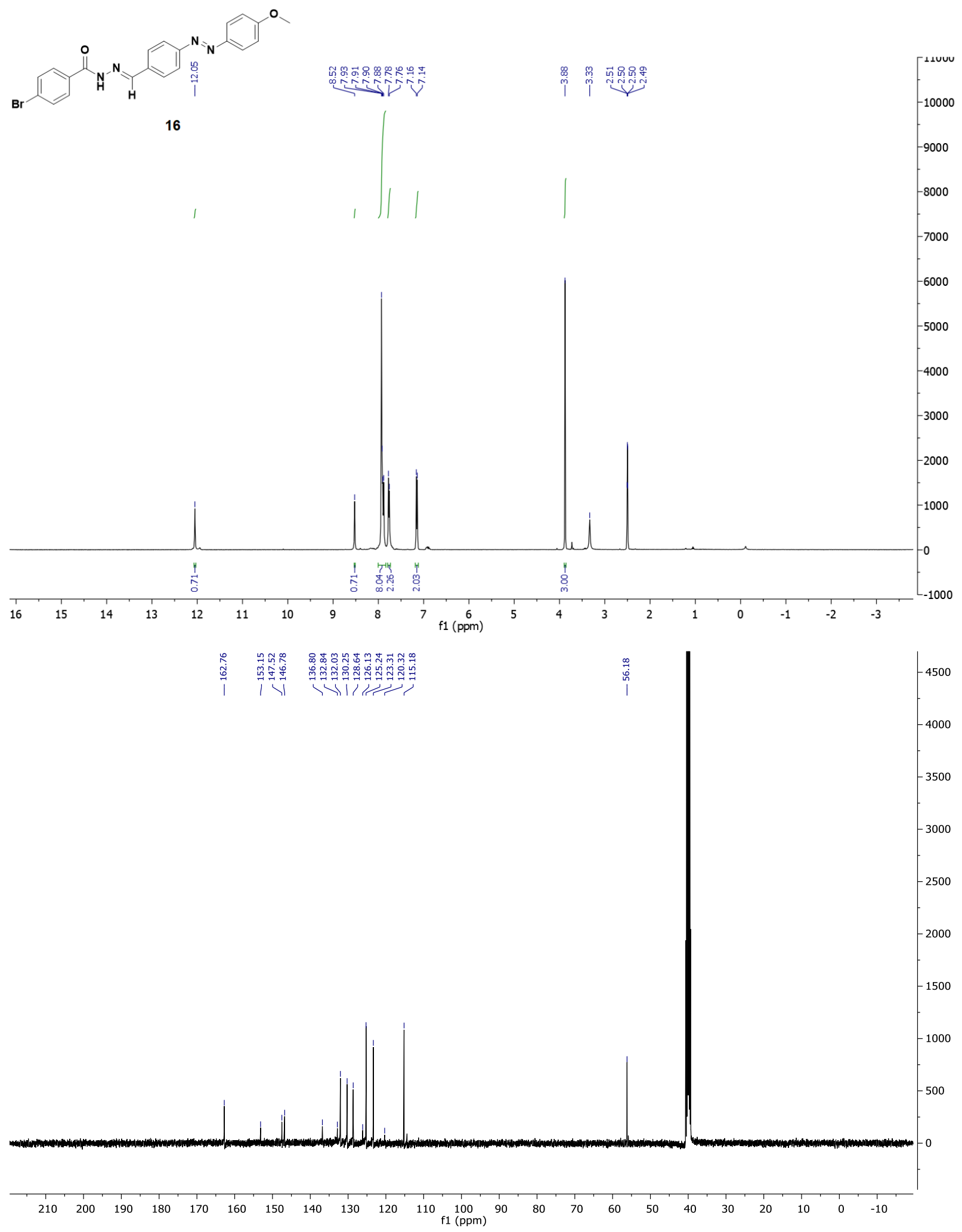



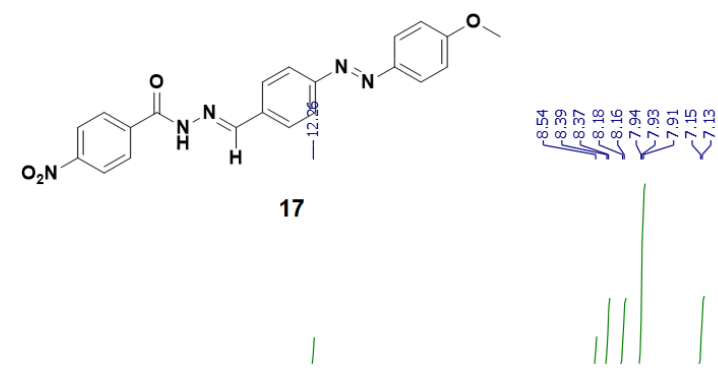

$-16000$

$-15000$

$-14000$

13000

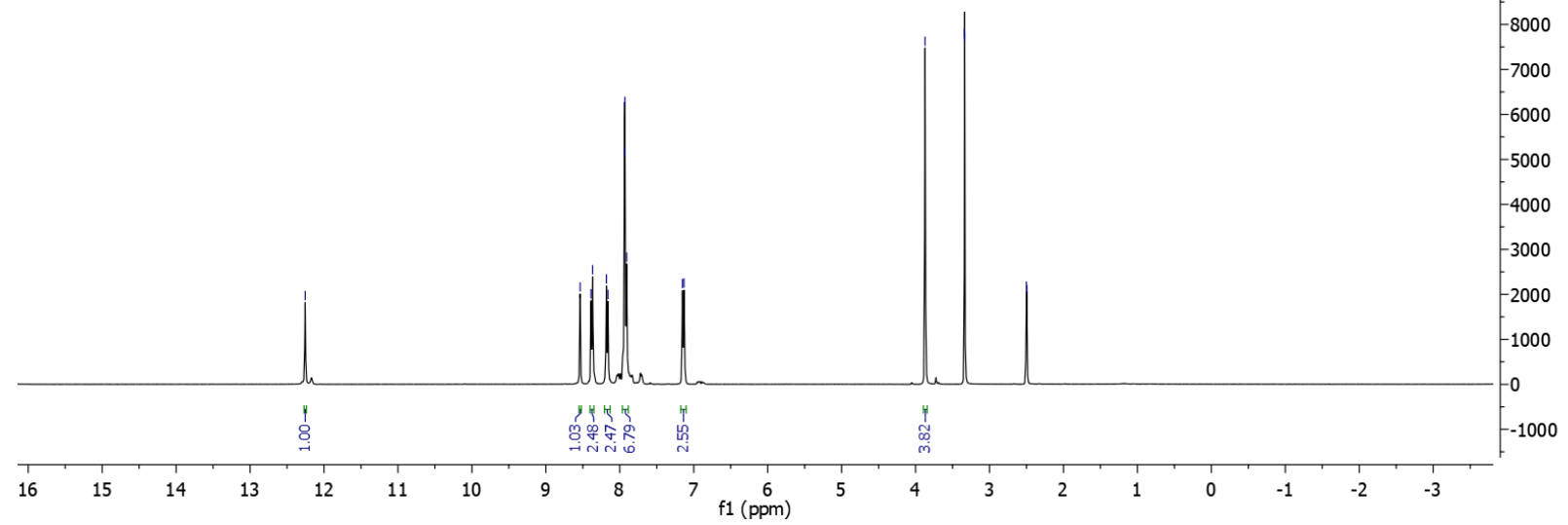

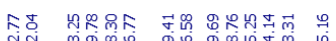

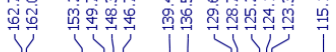

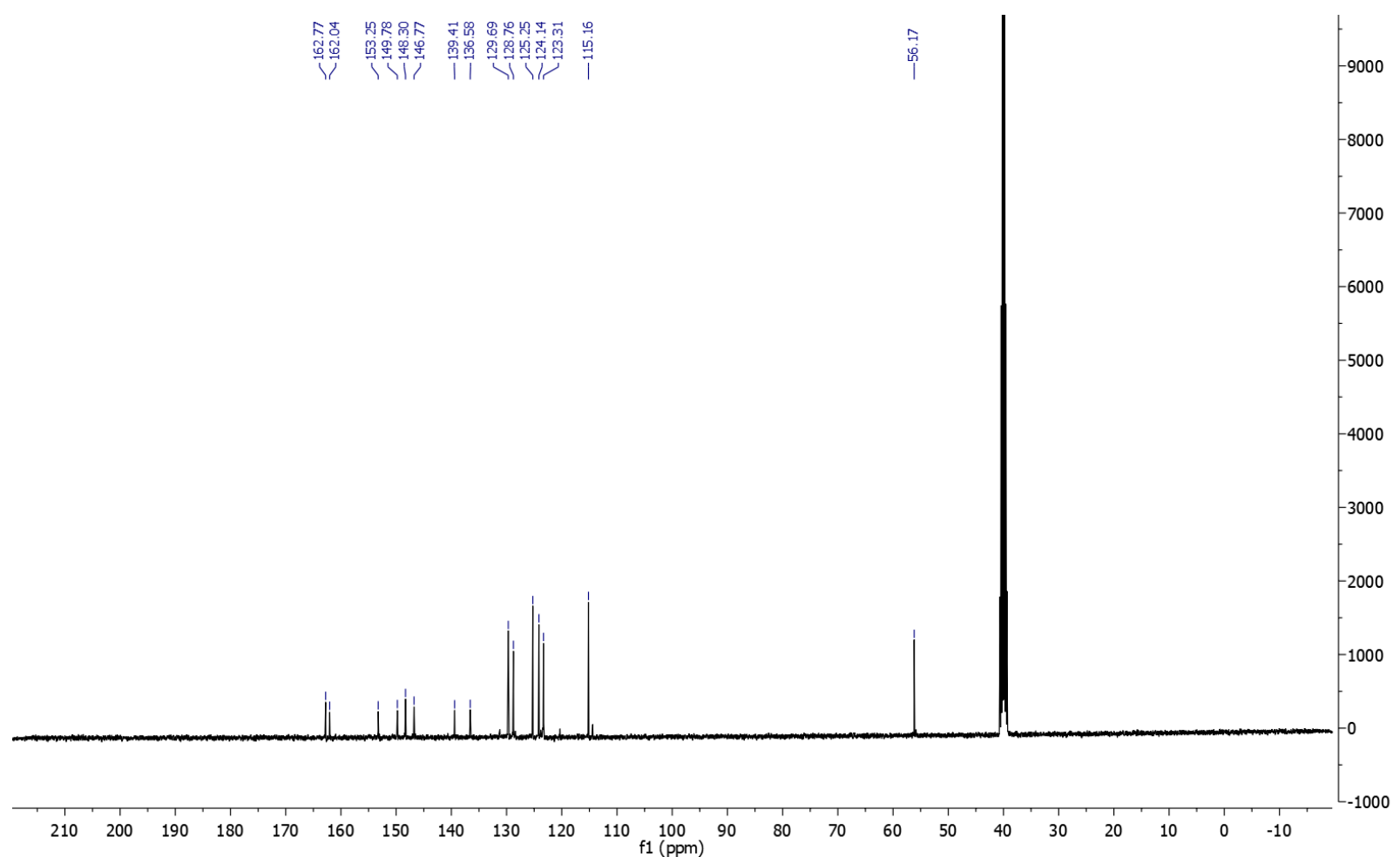




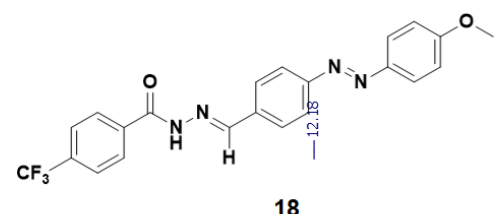

18

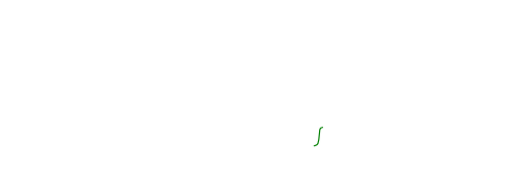

แับำ워

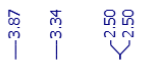
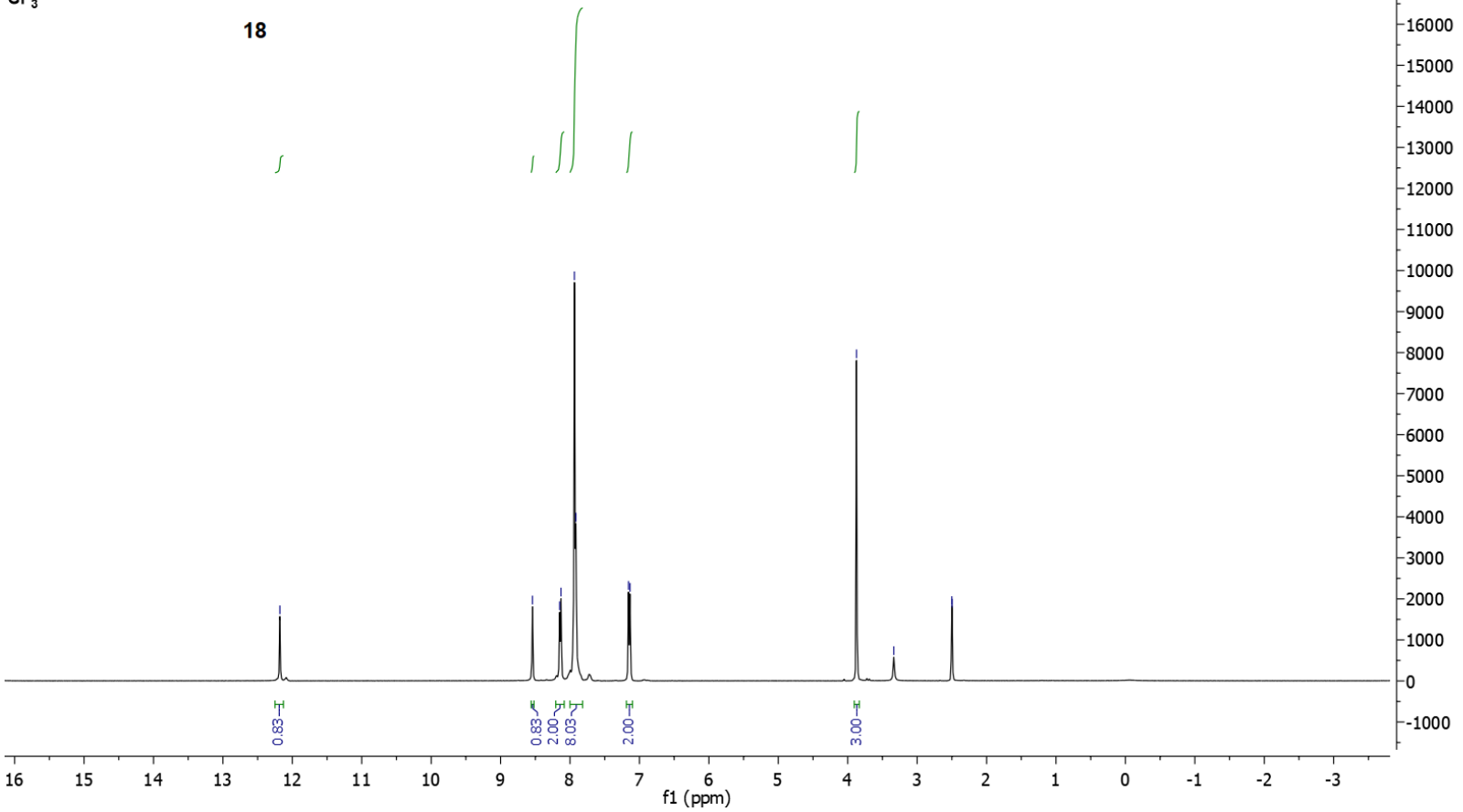

ำกำ ํํำ

V

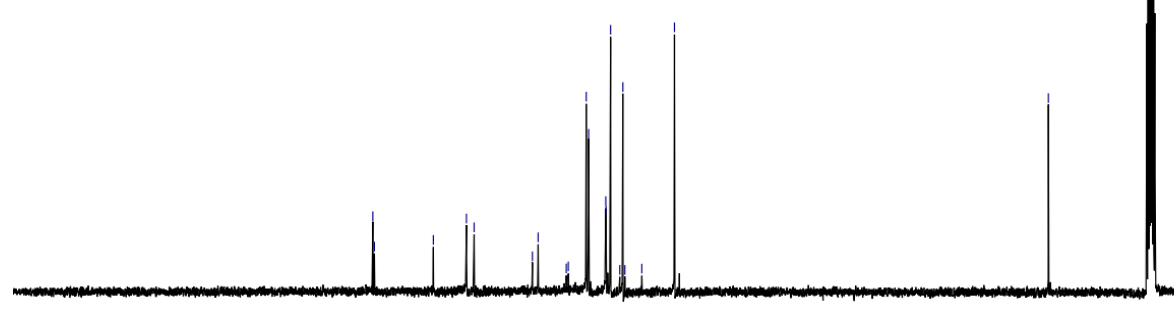

9
in
in

$\begin{array}{lllllllllll}210 & 200 & 190 & 180 & 170 & 160 & 150 & 140 & 130 & 120 & 110 \\ \mathrm{f} 1(\mathrm{ppm}) & 100\end{array}$ 

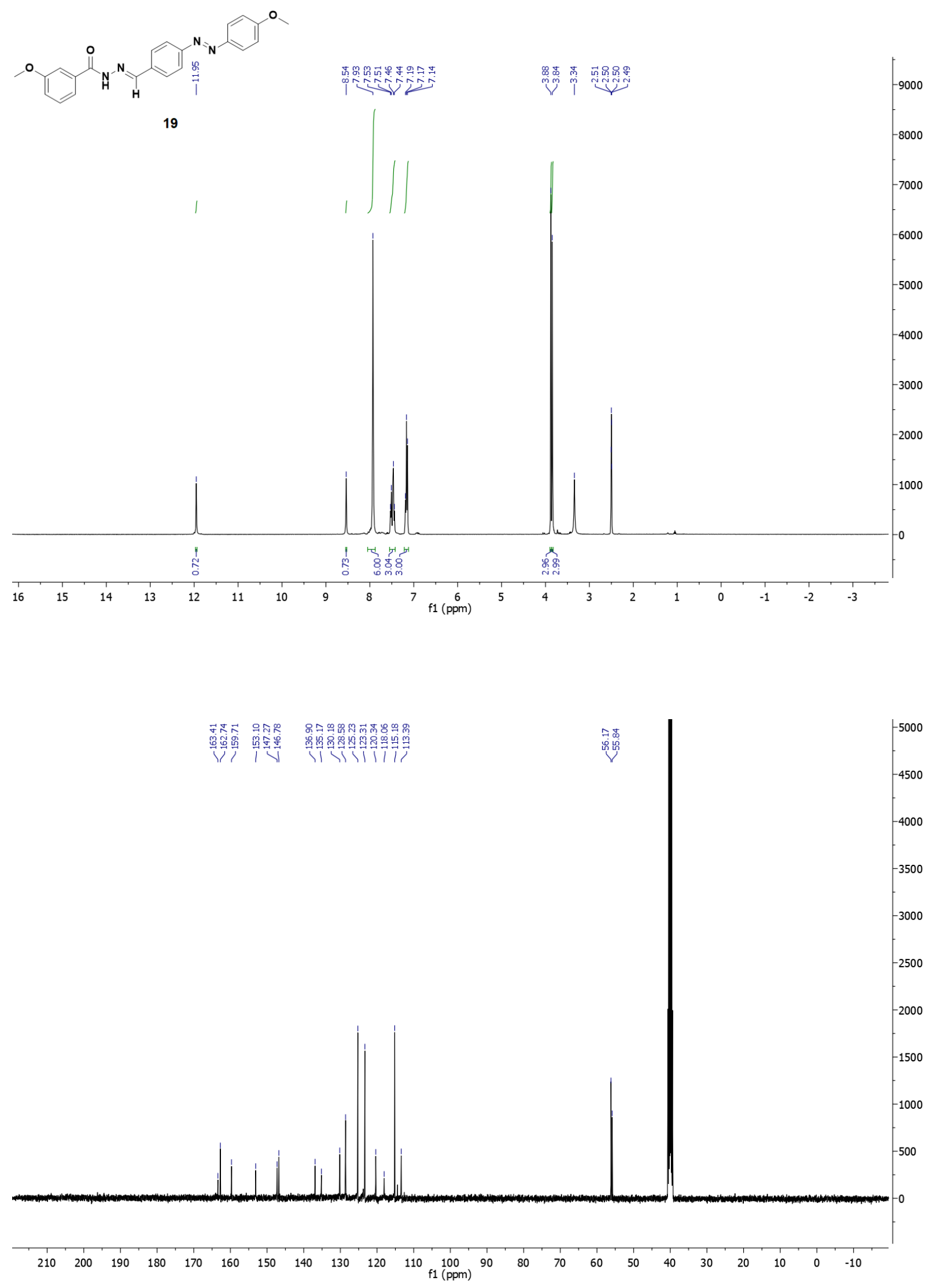


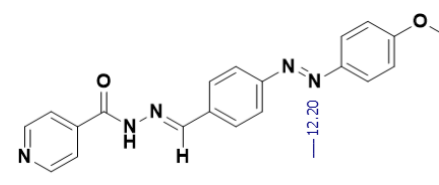

20

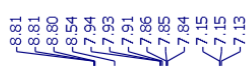

离
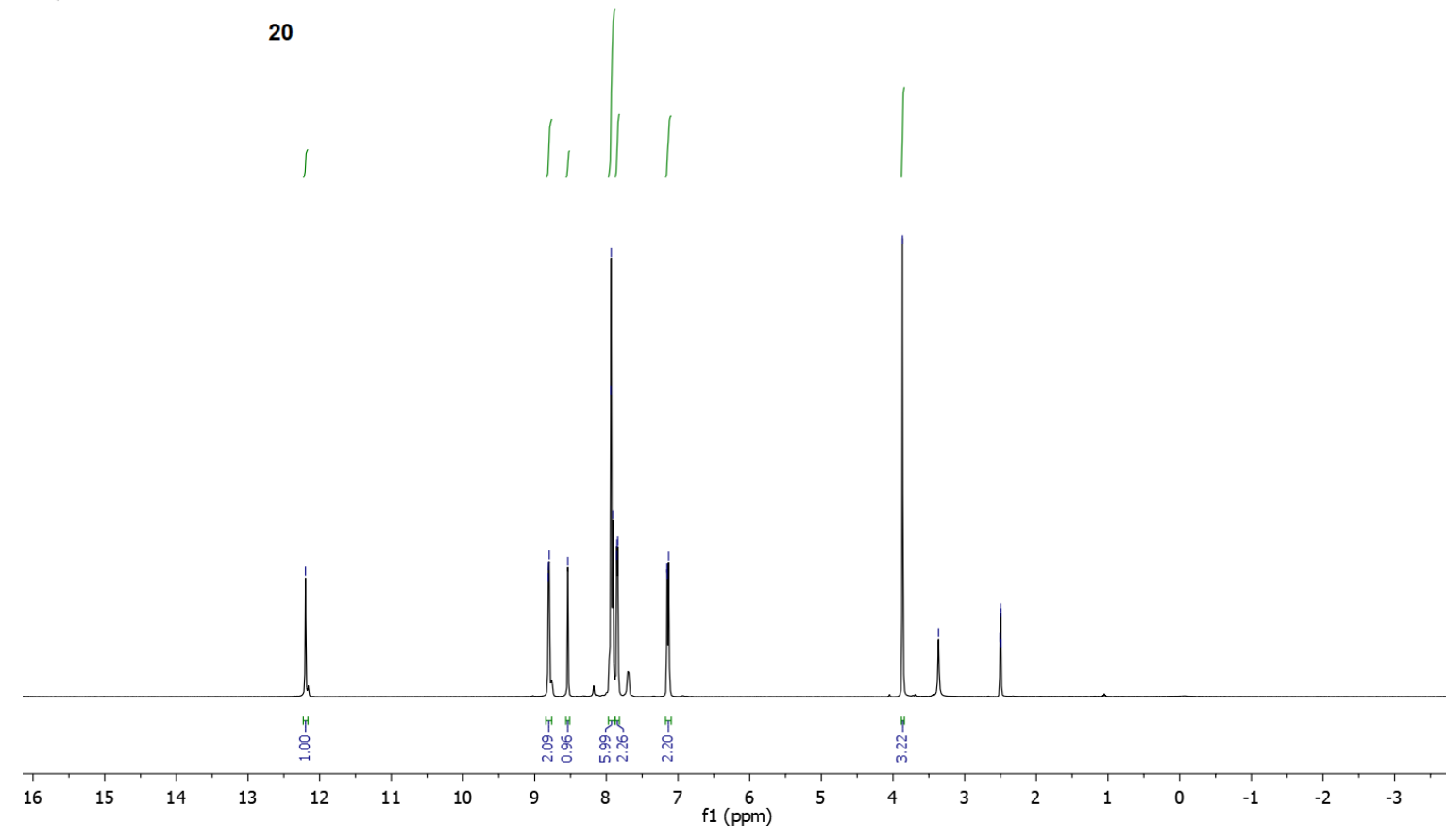

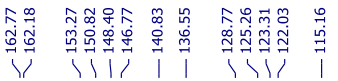

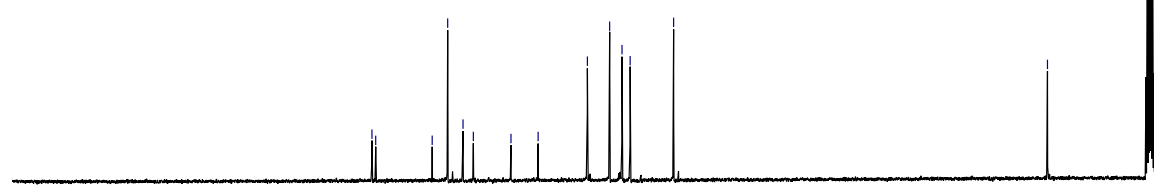

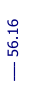

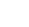

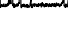

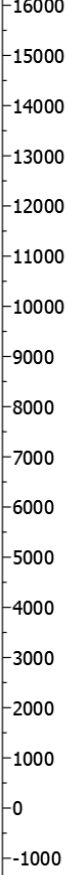

$-1000$

14000 


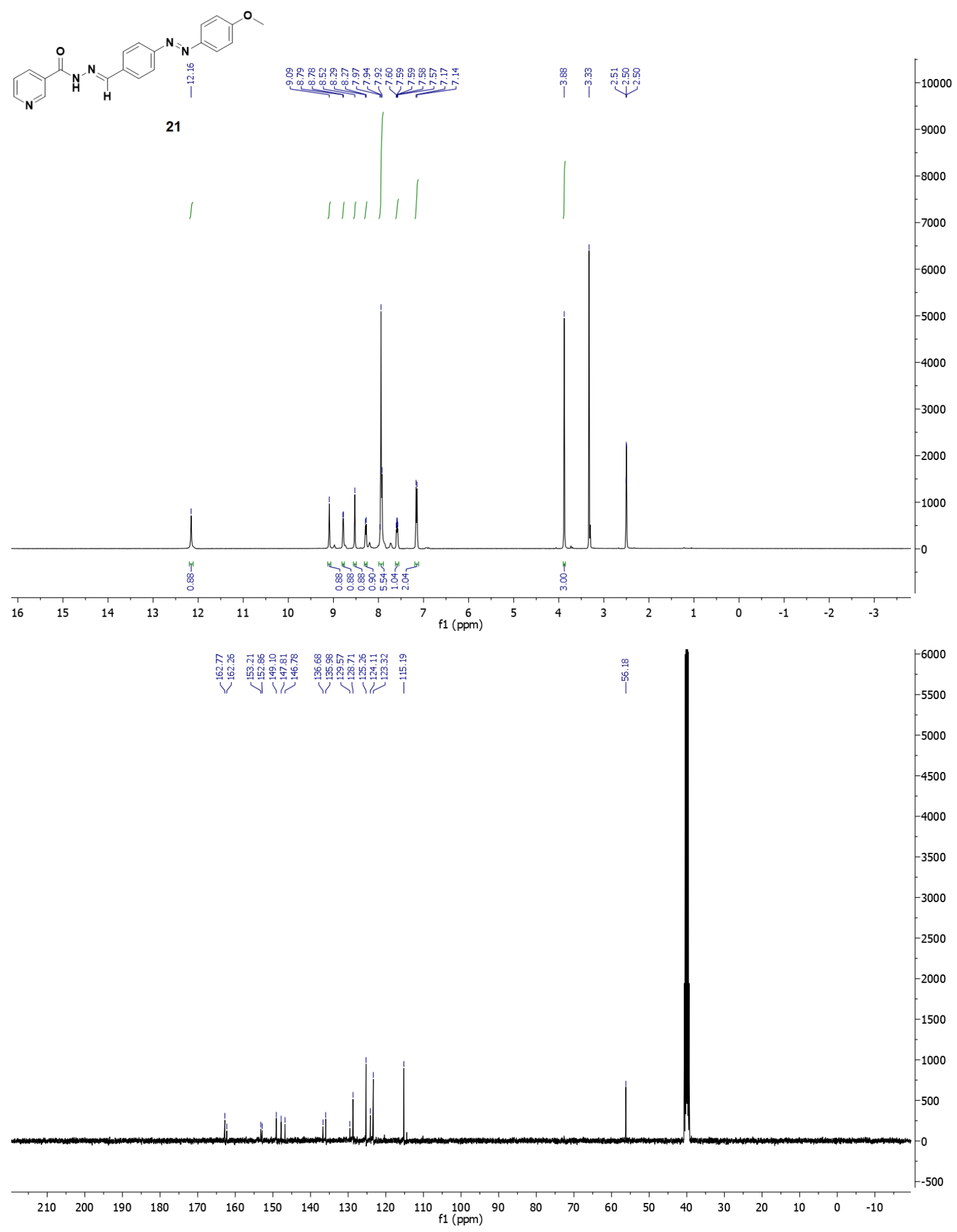




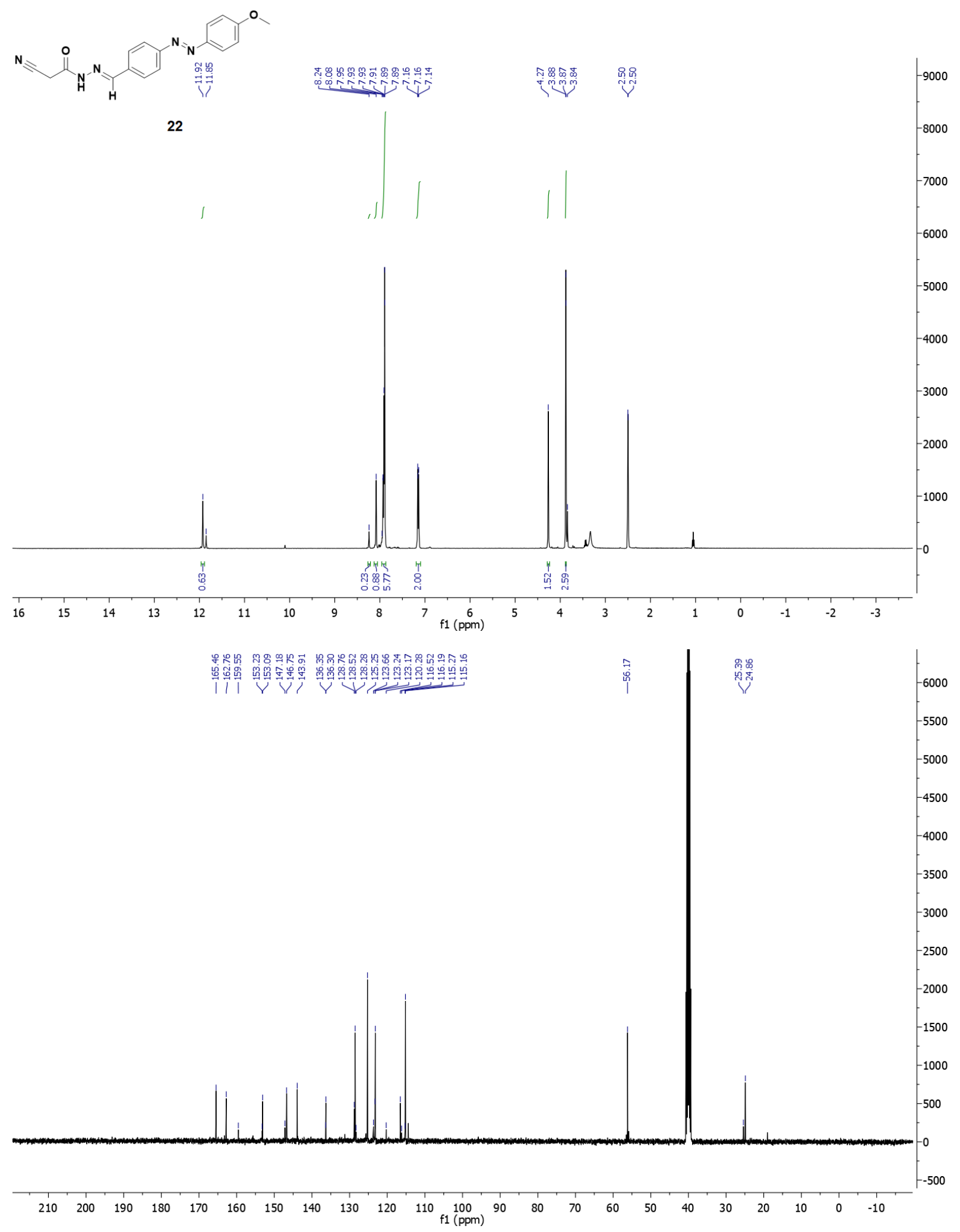




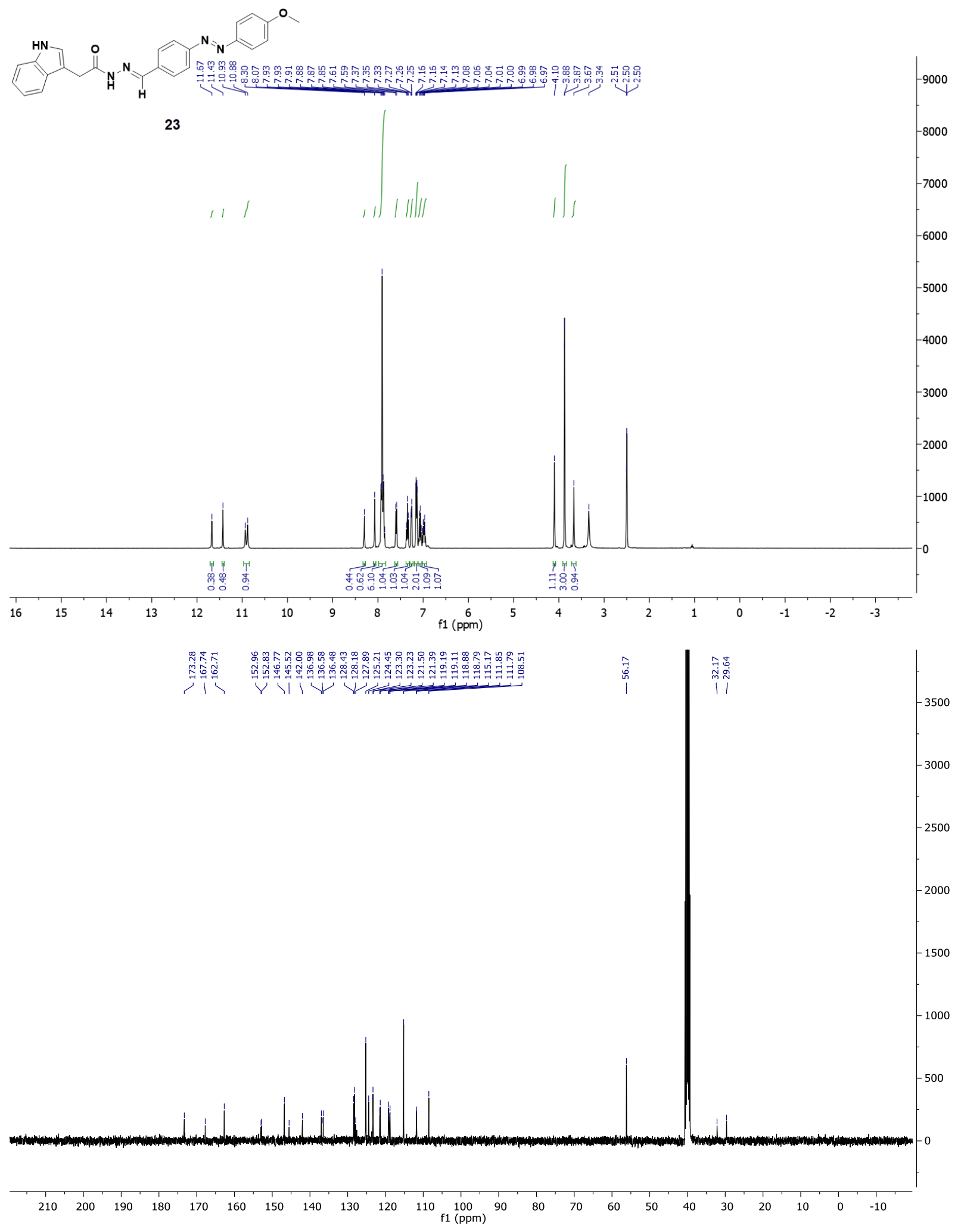




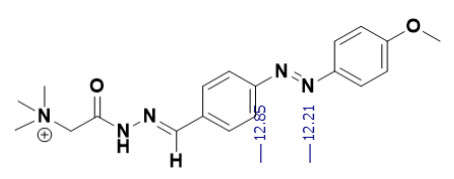

24
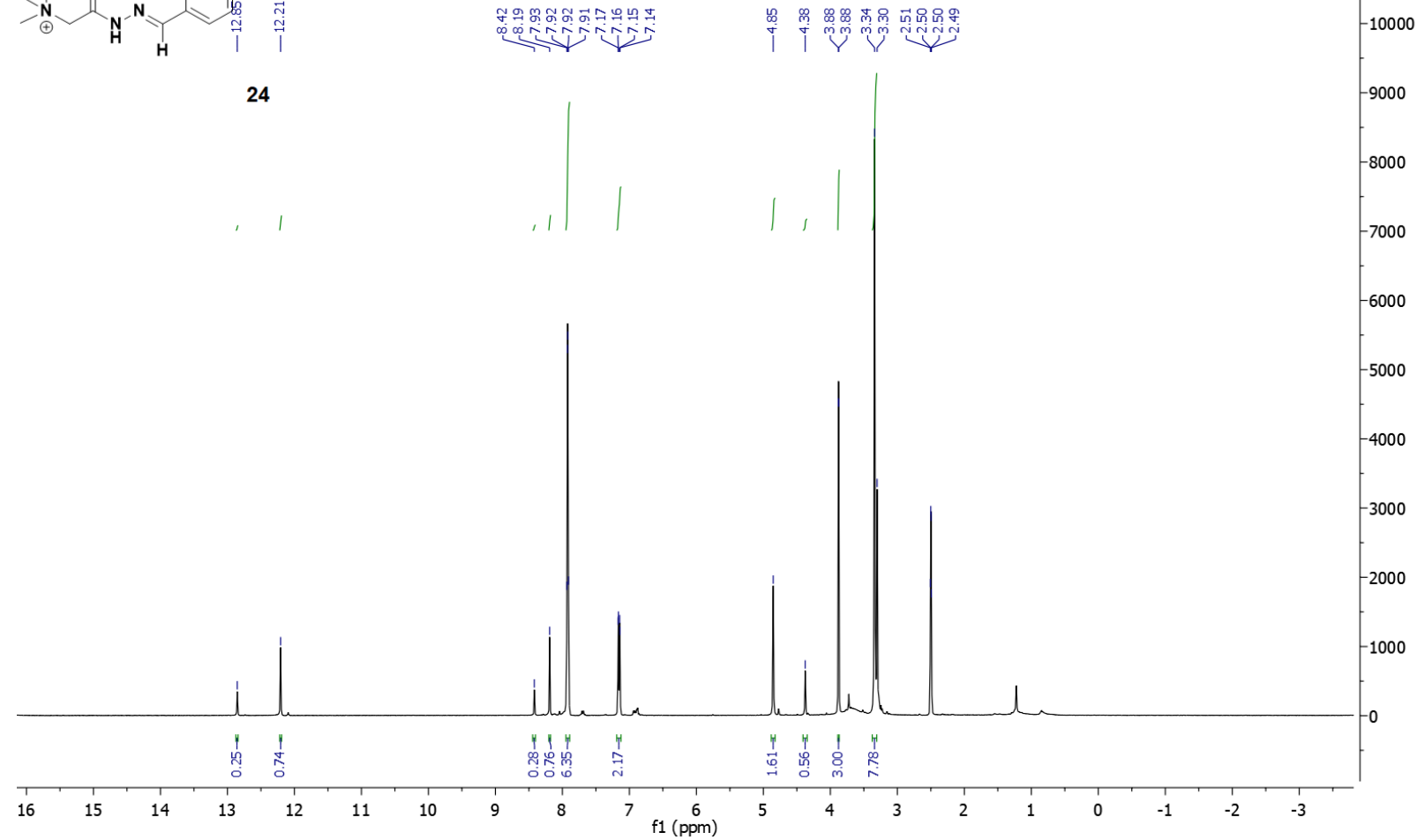

品

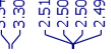

10000

000

000

000

000

000

000

落

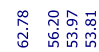

$-3500$

I \Y

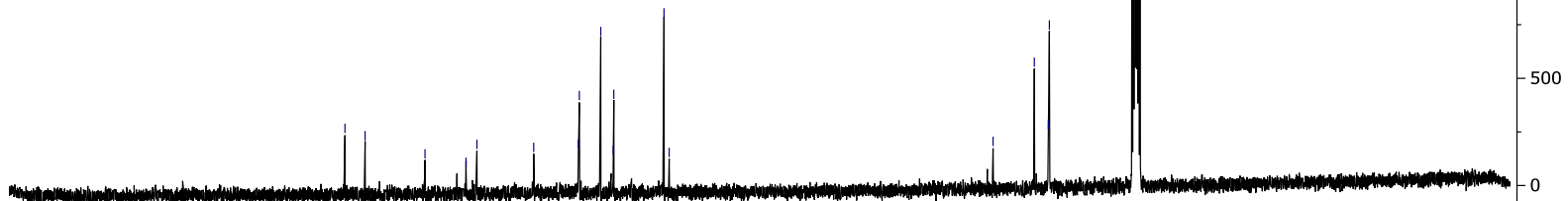




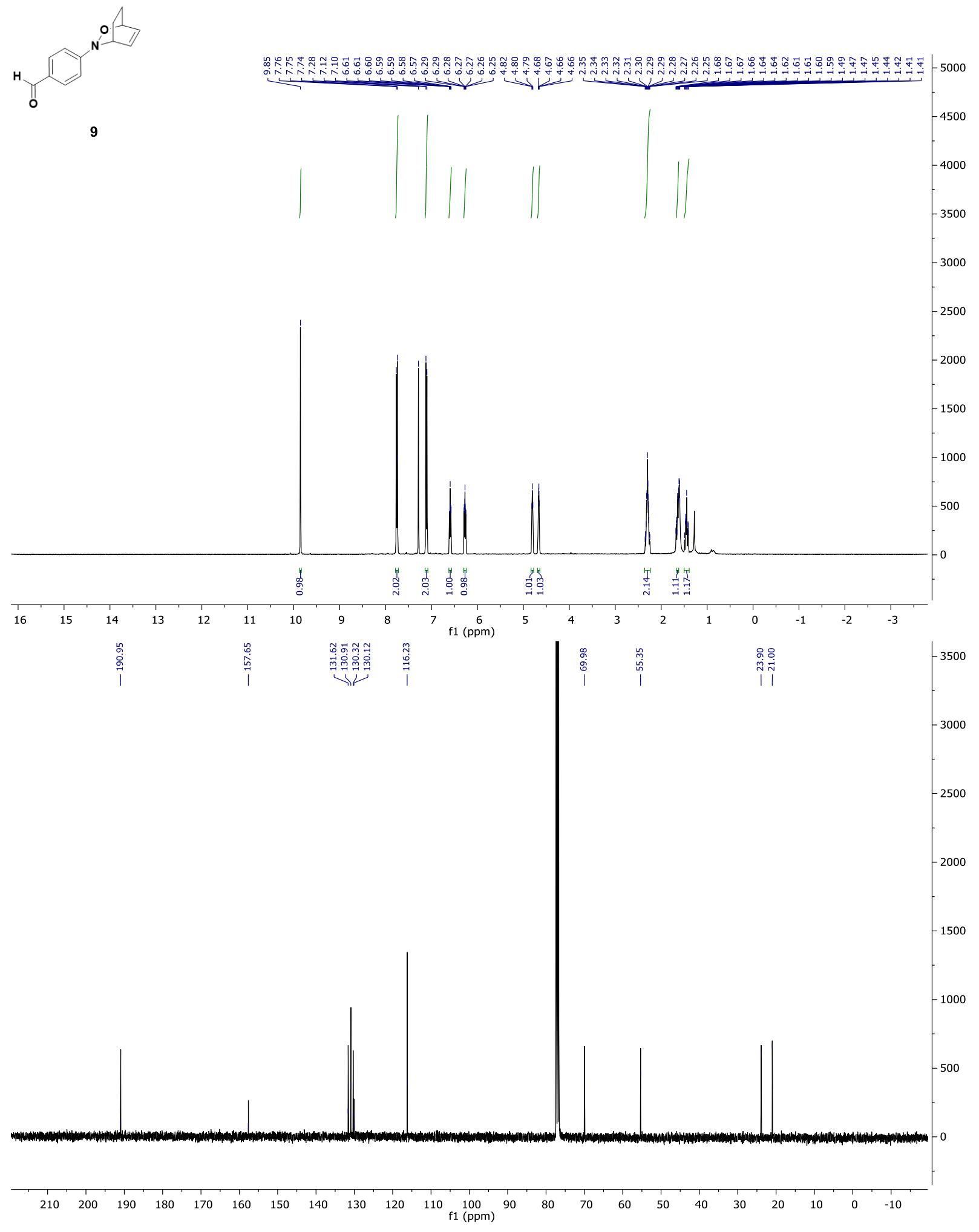




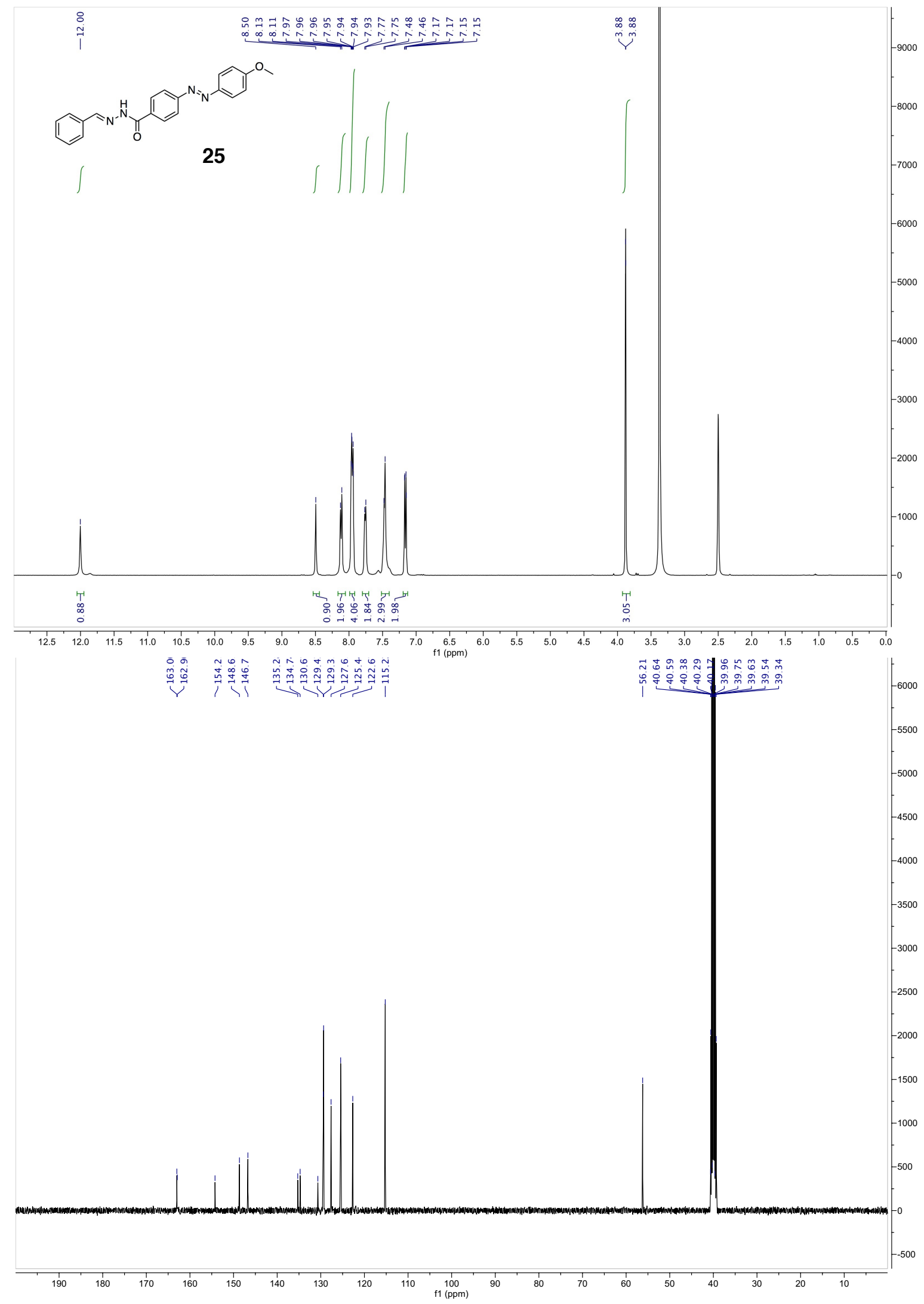

